\title{
Optimal Tunnel Coverage Problem by One New Nature-Inspired Energy Conservation Optimization
}

\author{
Jun Liu ${ }^{1,2 *}$ Tianyun Shi ${ }^{1}$, Ping $\mathrm{Li}^{1}$, Xuemei Ren ${ }^{2}$, Hongbin $\mathrm{Ma}^{2}$ \\ ${ }^{1}$ Institute of Computing Technologies, China Academy of Railway Sciences, Beijing, China \\ ${ }^{2}$ School of Automation, Beijing Institute of Technology, Beijing, China
}

Received 25 September 2014

Accepted 25 January 2015

\begin{abstract}
This paper mainly provides one new nature inspired Energy Conversation Optimization (ECO) method to search for the optimal sensor position of sensor network in the typical tunnels, fully covering the tunnel and dynamically locating the high-speed train in the tunnel. Firstly, one objective of this study is to briefly introduce the framework of transmitting the sensor's data in the railway network, the objectives and the constraints of the optimal coverage problem. Secondly, another objective of this paper is to provide some fundamental assumptions and concepts of ECO algorithm, together with the corresponding convergence analysis and the main steps of ECO algorithm. Additionally, the ECO algorithm is mainly utilized to address the optimal tunnel coverage problem. Numerical results mainly concentrate on the effectiveness of ECO algorithm comparing with the PSO algorithm and deal with four typical optimal coverage problems in the presence of the line tunnel, the indirect line tunnel and the complicated tunnels, etc. Finally, the ECO algorithm can offer the guideline for the smallest number of sensors and the corresponding positions in the given tunnel, fully covering the whole tunnel and providing the location of high-speed trains.
\end{abstract}

Keywords: Energy conversation optimization, optimal tunnel coverage problem, sensor network, railway transportation

\section{Introduction}

Comparing with other transportation methods, railway transportation has the advantages such as the huge transportation capacity, the high speed, the low transportation cost, the high safety and the low pollution, etc. Therefore, the China railway transportation plays a great role on the whole transportation in the presence of large distance for heavy lorry and small distance within 4 hours to 6 hours for the people. In the field of the modern railway transportation, the train location problem, which can be considered as one of the most interesting and important topics, is helpful for controlling the train's speed, reducing the interval time between two adjacent trains and improving the efficiency of railway transportation, etc. More importantly, it is also important to tackle with the train location problem under a variety of typical railways.

Recently, most of the currently and efficiently existing methods of train location $[1,2]$ on the basis of global position system (GPS) system and Beidou system (BDS) can provide the position service with high precision, finally leading to the huge transportation capacity. However, the GPS system or the BDS system probably cannot be applied in the tun-

\footnotetext{
*To whom all correspondences should be addressed. Email: DrJLBit@163.com or mathmhb@bit.edu.cn
} 
nels and the valley in the mountainous scenarios, since the corresponding high-speed trains cannot receive and respond to the signal from the satellite stations. In order to handle with the above-mentioned problem, we resort the sensor network or WiFi network to fully cover the whole tunnel, locating the train in the mountainous tunnel and dynamically controlling the train's speed. To find the optimal positions of sensors, swarm optimization methods possibly can be utilized to overcome this problem.

The original idea of those swarm optimization methods mainly mimics the mechanism in the nature or simulates the behavior of diverse swarm animals. More specifically, genetic algorithm (GA) [3], which is firstly formally introduced by John Holland in the 1970s, simulates the process of natural evolution and survival of the fittest by selection operator, crossover operator and mutation operator, etc. Particle swarm optimization (PSO) [4, 5], originally developed by Kennedy and Ebhert in 1995, simulates the movement of searching for food by the birds and the fish. In order to describe the idea of PSO algorithm, they introduce several new concepts including the particle, the particle's best previous position and the swarm's best previous position, etc. Ant colony optimization (ACO) [6], initially developed by Marco Dorigo in 1992, is inspired by swarm ants' behavior of seeking the path from the colony to the source of food and laying down pheromone trails. Simulated annealing (SA) [7] is initially described by Kirkpatrick in 1983 and the corresponding inspiration comes from the annealing of metallic molecule from high temperature to low temperature. Furthermore, there are also several novel optimization methods in recent years, such as firefly algorithm [8], cultural algorithms [9], Harmony search, intelligent water drops [10], charged system search [11], krill herd algorithm [12], artificial bee colony [13] and cuckoo search [14], etc.

In this contribution, one new Energy Conversation Optimization (ECO) method, which is firstly inspired by the law of energy conversation and particle swarm optimization $[15,16,17,18]$, is utilized to search for the optimal position of each sensor to cover the whole tunnel and provide the service of locating the train's position. Motivated by the law of energy conversation in the nature and other existing swarm optimization methods [19, 20, 21, 22, 23], several contributions in this paper can be highlighted as follows.

- A new nature-inspired optimization method, on the basis of particle swarm optimization and the law of energy conversation, is firstly developed to optimize the smallest number of sensors and search for the optimal position of each sensor.

- It is of importance to describe and model the objective function and the corresponding constraint of the optimal tunnel coverage problem.

- The convergence analysis of three mathematical models on ECO algorithm is helpful for parameter selection and the tradeoff between exploration ability and exploitation ability.

- Concerning the different typical tunnels in the mountainous scenarios, the ECO algorithm is employed to handle with the tunnel coverage problem, providing the optimal position of each sensor.

- The corresponding ECO method can be also applied for locating the people or the car in the realistic tunnel.

The remainder of this paper is organized as follows. Section 2 gives the description of the optimal converge tunnel problem in the presence of the single tunnel and the complicated tunnels. In order to deeply study the ECO algorithm, the new concepts, together with the corresponding convergence analysis, originate from the PSO algorithm and the law of energy conservation in Section 3. To tackle with optimal coverage tunnel problem by the ECO algorithm, the main steps of ECO algorithm are provided in Section 4. For the sake of the effectiveness of ECO algorithm, numerical results highlight that the ECO algorithm has the advantage over the PSO algorithm in Section 5. The ECO algorithm can search for the optimal position of each sensor in four complicated tunnels in Section 6. Section 7 concludes the important results and the future works of the optimal tunnel problem in the area of China's modern railway transportation. 


\section{Optimal Coverage Tunnel Problem}

\subsection{Background}

With the development of high-speed trains in China, the goal of modern railway transportation is to firstly ensure the safety of the trains, later increase the speed of the trains and increase the number of moving trains in one railway track. In order to achieve the aforementioned objectives, it is of necessity to utilize Beidou system (BDS) to locate the timevarying train position with high precision during the whole moving process, in addition, the Beidou system can be widely and successfully applied for the China's railway transportation in the future. However, the Beidou system or the GPS system probably cannot locate into the high-speed train in the context of the tunnels in the mountain and the valley in the mountain, and so on.

The problem of locating the train in the mountainous tunnel plays a great role on the safety and the efficiency of railway transportation in the presence of the China's west, since there exist a large number of the mountainous tunnels along the railway. For example, the corresponding well-known tunnels consist of Wuqiaolin tunnel, Dayaoshan tunnel and Jundushan tunnel, etc. Particularly, there are a lot of the mountainous tunnels in the railway from Cheng Du city to Kun Ming city, from Bao Ji city to Guang Yuan city and from Xi An city to An Kang city, etc. Therefore, the dynamical locating of highspeed train in the mountainous tunnel is one of the important and interesting topics in the field of the current and future modern railway transportation.

In order to tackle with this problem, the sensor network can provide the time-varying position of high-speed train with high precision in the given tunnel. The framework of transmitting sensor data is depicted in Fig. 1. Firstly, when the high-speed train goes into the tunnel and the signal is transmitted between the train and the sensor, providing the train's position in the tunnel. Secondly, with the help of other sensors in the tunnel, the position of high-speed train is transmitted to the station and data center via the GSM-R railway network. Thirdly, the data including the position of each train are greatly helpful for controlling the speed of those trains in the railway and optimizing the train's scheduling table, in addition, those corresponding data to be obtained also benefit the three-dimensional visualizing, data analysis and data mining.

\subsection{Objectives and Constraints of Optimal Coverage Tunnel Problem}

In order to deeply analyze the optimal coverage tunnel problem, the main task of this subsection is to introduce the description of the fundamental concepts on optimal coverage tunnel problem, together with the objectives and the corresponding constraints.

Definition 1 Given the center point $C_{i}\left(x_{i}, y_{i}\right)$ and the radius $R_{i}$ of the ith sensor, the coverage region $S_{i}$, which is composed of the discrete points $(x, y)$ in the two-dimensional space, can be mathematically defined as

$$
\left(x-x_{i}\right)^{2}+\left(y-y_{i}\right)^{2} \leqslant R_{i}^{2} .
$$

In the three dimensional space, the coverage region $S_{i}$ can be mathematically expressed by

$$
\left(x-x_{i}\right)^{2}+\left(y-y_{i}\right)^{2}+\left(z-z_{i}\right)^{2} \leqslant R_{i}^{2} .
$$

Definition 2 Given the sensor network with n nodes and its coverage region $S_{i}(1 \leqslant i \leqslant n)$, the coverage region $\Gamma$ of sensor network is

$$
\Gamma=S_{1} \cup S_{2} \cup S_{3} \cup \cdots \cup S_{n}
$$

where the center point $C_{i}$ denotes $\left(x_{i}, y_{i}, z_{i}\right)$ in the three dimensional space.

Given one whole region $\Omega$, the sufficient condition of the full coverage by one sensor network can be mathematically expressed by

$$
\Omega \subseteq \Gamma .
$$

Definition 3 The optimal coverage problem is to subject to the condition of (4) and minimize the following equation.

$$
\min \Gamma-\Omega
$$

Definition 4 In this paper, two typical tunnels to be considered are composed of the line tunnel and the complicated tunnel. 


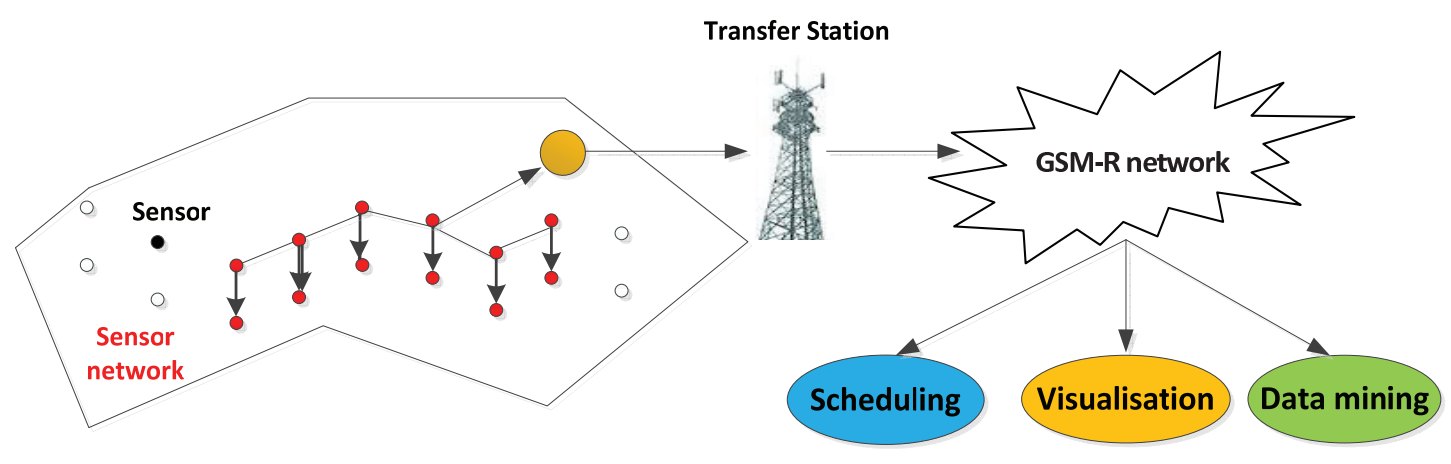

Figure 1: Framework of transmitting data in the sensor network

Concerning the line tunnel, the optimal tunnel coverage problem by the sensor network in the presence of the constraint condition (4) can be mathematically expressed by

$$
\min \sum_{i=1}^{n-1}\left[C_{i}+R_{i}-\left(C_{i+1}-R_{i+1}\right)\right] .
$$

In the different complicated tunnels, it is so difficult to set up its mathematical model of the complicated tunnel. In order to handle with this problem, the complicated tunnel $L$ is composed of several discrete order points, so the corresponding tunnel $L$ can be mathematically described by

$$
L=\left\{\left(x_{1}, y_{1}\right),\left(x_{2}, y_{2}\right),\left(x_{3}, y_{3}\right), \cdots,\left(x_{m}, y_{m}\right)\right\}
$$

where $m$ denotes the number of discrete points along the tunnel.

The considered objective function of the optimal tunnel coverage problem can be mathematically described by

$$
\max \sum_{i=1}^{m} f_{i}(x)-\min \sum_{i=1}^{m} g_{i}(x)
$$

where $f_{i}(x)$ is the number of discrete points only covered by single sensor, and $g_{i}(x)$ is the number of discrete points simultaneously covered by two sensors or more sensors.

In order to achieve the goal of the optimal coverage tunnels including the line tunnel and the complicated tunnel, it is of importance to search for the center points of each sensor in sensor network. Therefore, the task of the following sections is to introduce the new swarm optimization method to find the optimal center of every sensor, leading to fully and optimally covering the tunnel.

\section{Energy Conversation Optimization}

By the inspiration of the law of energy conservation and several new swarm optimization methods, this section is mainly to incorporate the original idea in Physics into swarm optimization in evolutionary computation to possibly reduce the risk of premature convergence. Several basic concepts and definitions, corresponding to the incorporation between the law of energy conservation and swarm optimization, are introduced to deeply understand the essence and main steps of Energy Conversation Optimization (ECO) algorithm.

\subsection{Basic Concepts and Definitions in ECO Algorithm}

To better illustrate the ECO algorithm, we firstly introduce the basic assumptions and notations on the law of energy conversation and evolutionary computation.

Definition 5 Consider the mass $M$ and the current velocity $v$ of the solution. Power energy $P_{f}$ of one solution, mainly depending on its mass and the velocity, is physically defined by the following equation.

$$
P_{f}=\frac{1}{2} M v^{2}
$$

Definition 6 Consider the objective function $f$ and the tth best position $G(t)$. The height $\Delta h(t+1)$ in the 
ECO algorithm physically denotes the changed objective fitness. Taking the minimization problem into account, the height $\Delta h(t+1)$ is

$$
\Delta h(t+1)=\left\{\begin{array}{cl}
f(G(t))-f(G(t+1)) & f(G(t+1))<f(G(t)) \\
0 & f(G(t+1))=f(G(t))
\end{array}\right.
$$

where $t$ denotes the number of current generation.

In the context of the maximization problem, the corresponding height $\Delta h(t+1)$ is

$$
\Delta h(t+1)=\left\{\begin{array}{cl}
f(G(t+1))-f(G(t)) & f(G(t+1))>f(G(t)) \\
0 & f(G(t+1))=f(G(t)) .
\end{array}\right.
$$

Therefore, potential energy $P_{e}$ of one solution is defined as

$$
P_{e}(t)=M g \triangle h(t)
$$

where g, generally setting to 9.8, denotes the gravitational acceleration in Physics.

Lemma 1 Consider the resistance force $f$ and the distance s along the trajectory from point A to point B. As illustrated in Fig. 2, the current velocities at point $A$ and point $B$ are assumed to be $v_{1}$ and $v_{2}$, respectively. According to the law of energy conversation, current energy at point A, including potential energy and power energy, is equal to the energy at point $B$, including current power energy and the damping energy. The law of energy conversation can be mathematically described by

$$
M g \triangle h+\frac{1}{2} M v_{1}^{2}=\frac{1}{2} M v_{2}^{2}+f s
$$

where the first part and the second part in the left hand side of (13) denote the potential energy and power energy at A point, respectively. In addition, the first part and the second part in the right hand side of hand side of (13) denote the power energy at $B$ point and the additional energy, which overcomes the friction force and the resistance force from point $A$ to point $B$, respectively.

Lemma 2 Consider the whole process regarding energy conversation, the whole energy, including power energy and potential energy, at the first step is equal to the energy of resistance force during the whole evolutionary process when one solution only searches for the suboptimal optimum. Mathematically speaking, energy conversion in the whole evolutionary process can be described by

$$
M g h_{f}+\frac{1}{2} M v_{f}^{2}=M g h_{u}+\sum_{i=1}^{\infty} f_{i} s_{i}
$$

where $h_{f}$ and $v_{f}$ denote the initial objective fitness and the velocity of one solution. And $h_{u}\left(h_{u} \leqslant h_{f}\right)$ is the final objective fitness at the last step, while $f_{i}$ and $s_{i}$ denote the resistance force and the moving distance at the ith step, respectively.

Ideally speaking, if the solution in ECO algorithm searches for the global optimum, the final objective fitness $h_{u}$ equals to 0 . That is to say, the equation (14) can be mathematically rewritten as

$$
M g h_{f}+\frac{1}{2} M v_{f}^{2}=\sum_{i=1}^{\infty} f_{i} s_{i}
$$

\subsection{Two Difficult Problems in Mathematical Model}

In order to incorporate the law of energy conversation and swarm optimization, it is of importance to handle with two typical problems which are closely related to the resistance energy and the conversion from potential energy to power energy in different landscapes of objective functions.

Firstly, it is hard to implement the conversation from one point to another point, since the resistent force $f$ and the distance $s$ from point A to point B are difficultly calculated and obtained during the evolutionary process. In order to cope with the abovementioned problem, the damping factor $\alpha$ is introduced to overcome the corresponding complex problem, and the second part in the right hand side of (13) is considered to be one part of the current power energy. Hence, the resistance force energy can be roughly expressed by

$$
f s=\alpha \times \frac{1}{2} M v_{2}^{2}
$$

where $\alpha(\alpha>0)$ is the damping factor of the system.

According to (16), the equation of (13) can be mathematically rewritten as

$$
M g \triangle h+\frac{1}{2} M v_{1}^{2}=\frac{1}{2} M v_{2}^{2}(1+\alpha) .
$$




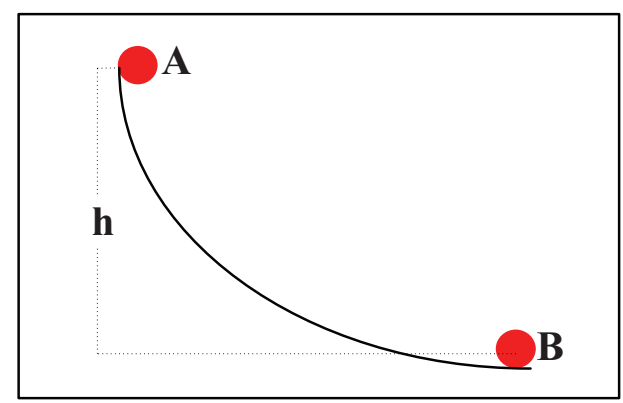

Figure 2: The process from potential energy to power energy

Therefore, the current velocity $v_{2}$ can be mathematically expressed by

$$
v_{2}=\sqrt{\frac{2 g \triangle h+v_{1}^{2}}{1+\alpha}} .
$$

Secondly, in the case of different landscapes and different ranges of solution space, the height $h$ in the landscape of some objective functions may be very high, resulting in large velocity of each solution. However, the height $h$ in the landscape of other functions may be very low, resulting in small velocity. To address this complex problem, one factor $\beta$ between power energy and potential energy is introduced to ensure that potential energy in different landscapes can give rise to suitable velocity of each solution. In order to achieve this goal, the initial potential energy of each solution is equally assumed to be the maximum power energy, and it can be mathematically expressed by

$$
\frac{1}{2} M v_{\max }^{2}=\beta M g h_{\max }
$$

where $v_{\max }$ and $h_{\max }$ are the maximum velocity and the initial objective fitness in the evolutionary process, respectively.

According to (19), the interchanged factor $\beta$ from potential energy to power energy can be mathematically calculated by

$$
\beta=\frac{v_{\max }^{2}}{2 g h_{\max }} .
$$

In terms of (18) and (20), the current velocity $v_{2}$ at point $\mathrm{B}$ can be also rewritten as

$$
v_{2}=\sqrt{\frac{2 \beta g \Delta h+v_{1}^{2}}{1+\alpha}} .
$$

In summary, the damping factor $\alpha$ in (21) essentially controls the convergence speed of one solution, while another factor $\beta$ is to ensure that the ECO algorithm can be adaptively utilized in different practical objective functions. The goal of introducing the law of energy conversation in evolutionary process is to increase current velocity of each solution, possibly getting away from the local optimum.

\subsection{Swarm Topology of ECO Algorithm}

The topology of all solutions, which essentially controls the speed of spreading the location of global optimum, plays a great role on high efficiency and the analysis on swarm behavior. As is well known, the high connected topology can give rise to large convergence speed, resulting in low computation time and premature convergence, while the low connected topology leads to small convergence speed, leading to too much computational time and high successful ratio finding good solution. Therefore, it is of importance to select suitable topology of all solutions in the practical problem. From the perspective of evolutionary method, swarm topology mainly plays a great role on the attractor of each solution in the ECO system. 


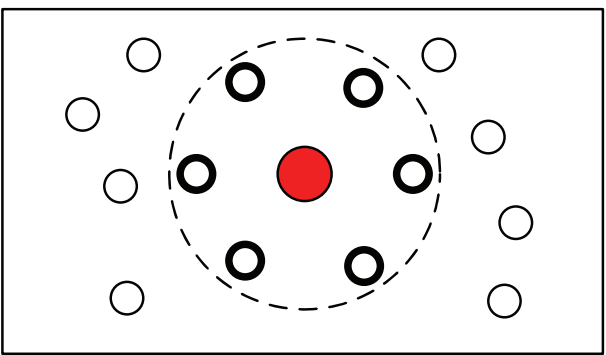

Figure 3: The topology of group animals in the nature

Swarm topology in ECO algorithm is mainly inspired by the behavior of many group animals in the nature. Specifically speaking, a kind of group animals do not have the communication with all animals, however, they merely have connection with its neighbors $[24,25]$. From the graph viewpoint, the corresponding topology is depicted in Fig. 3.

Compared with other evolutionary algorithms, ECO algorithm has the advantages over the mathematical analysis from the perspective of swarm topology. In order to conduct mathematical analysis of ECO algorithm, Laplacian matrix and the corresponding eigenvalue are introduced to analyze the connected density and the connectivity, respectively. In addition, the connected density mainly plays a great role on the speed of spreading the global optimum and the corresponding convergence rate, while the connectivity chiefly determines whether all solutions can converge into the global optimum or suboptimal optimum or not.

In Graph, there are two typical sets which are composed of the vertex set $N$ and the edge set $E$. The vertex set $N$ consists of $n$ nodes, such as $\left\{N_{1}\right.$, $\left.N_{2}, \cdots, N_{n}\right\}$, and the edge set $\mathrm{E}=\left\{e_{i j}, \mathrm{i}=1,2, \cdots, \mathrm{n}\right.$; $\mathrm{j}=1,2, \cdots, \mathrm{n}\}$ denotes the topology of the swarm. In addition, the element $e_{i j}$ in the edge set $E$ is defined as

$$
e_{i j}= \begin{cases}0 & N_{i} \text { cannot connect with } N_{j} \\ 1 & \text { Otherwise }\end{cases}
$$

where $N_{i}$ and $N_{j}$ denote the starting node and the ending node, respectively.

According to swarm topology together with the corresponding edge set, the adjacency matrix $E$ can be mathematically expressed by

$$
E=\left[\begin{array}{ccccc}
e_{11} & e_{12} & e_{13} & \cdots & e_{1 n} \\
e_{21} & e_{22} & e_{23} & \cdots & e_{2 n} \\
e_{31} & e_{32} & e_{33} & \cdots & e_{3 n} \\
\cdot & \cdot & \cdot & \cdots & \cdot \\
e_{n 1} & e_{n 2} & e_{n 3} & \cdots & e_{n n}
\end{array}\right]_{n \times n}
$$

where $e_{i j}$ denotes the connected edge between node $N_{i}$ and node $N_{j}$.

On the basis of adjacency matrix $E$, Laplacian matrix $L$, which is also called the admittance matrix or Kirchhoff matrix, can be mathematically expressed as

$$
L=\left[\begin{array}{ccccc}
l_{11} & l_{12} & l_{13} & \cdots & l_{1 n} \\
l_{21} & l_{22} & l_{23} & \cdots & l_{2 n} \\
l_{31} & l_{32} & l_{33} & \cdots & l_{3 n} \\
\cdot & \cdot & \cdot & \cdots & \cdot \\
l_{n 1} & l_{n 2} & l_{n 3} & \cdots & l_{n n}
\end{array}\right]_{n \times n}
$$

where the element $l_{i j}$ in Laplacian matrix $L$ is

$$
l_{i j}=\left\{\begin{array}{cc}
-e_{i j} & i \neq j \\
\sum_{j} e_{i j} & i=j .
\end{array}\right.
$$

Furthermore, all eigenvalues of Laplacian matrix $L$ can be sorted by

$$
0=\lambda_{1} \leqslant \lambda_{2} \leqslant \cdots \leqslant \lambda_{i} \leqslant \cdots \lambda_{n}
$$

where $\lambda_{i}$ denotes the $i$-th sorted eigenvalue of Laplacian matrix.

According to Laplacian matrix and its eigenvalues, several conclusions and remarks can be summarized as 
1. If the graph of one topology is an unconnected graph, the second smallest eigenvalue $\lambda_{2}$ is equal to 0 . In addition, $\lambda_{2}$ can determine whether the graph of the topology is the connected graph or not.

2. According to the basic property of Laplacian matrix, Laplacian matrix is positivesemidefinite. And the first smallest eigenvalue $\lambda_{1}$ strictly equals to 0 and its eigenvector is [1, $1,1, \cdots, 1]$. Others eigenvalues in matrix $L$ are strictly larger than 0 under the connected graph.

3. Particularly, the second smallest eigenvalue $\lambda_{2}$ can mathematically denote the connected density of swarm topology.

4. The main diagonal of Laplacian matrix can express the connected density of swarm topology.

In order to achieve the convergence of whole solution, its topology in the ECO algorithm is selected to be the connected topology, whose second smallest eigenvalue $\lambda_{2}$ is strictly larger than 0 . In addition, the parameter $\lambda_{2}$ physically denotes the speed of interchanging the valuable information of the global optimum.

\subsection{Convergence Analysis for the ECO Algorithm}

In the case of the ECO algorithm, the velocity and the position of each solution, under the assumption of $\gamma(t)=\frac{2 \beta g \Delta h}{v^{2}}$, can be mathematically described by

$$
\begin{gathered}
v(t+1)=\sqrt{\frac{1+\gamma(t)}{1+\alpha}} v(t)+\sum_{k=1}^{n} c_{k} r_{k} e_{k}\left(G_{k}(t)-x(t)\right) \\
x(t+1)=v(t+1)+x(t)
\end{gathered}
$$

where $c_{i}$ denotes the constant variable and $r_{k}$ denotes the random variable in $[0,1]$. Additionally, $e_{k}$ is the topology of all particles at $t$ th step.
Assuming $\varphi_{j}(t)=c_{j} r_{j} e_{j}$, the equations of (27) and (28), can be simply rewritten as

$$
\begin{gathered}
v(t+1)=\sqrt{\frac{1+\gamma(t)}{1+\alpha}} v(t)+\sum_{j=1}^{n} \varphi_{j}(t)\left(G_{j}(t)-x(t)\right) \\
x(t+1)=v(t+1)+x(t) .
\end{gathered}
$$

Furthermore, (29) at the (t+1)th step can also be described by

$$
\begin{gathered}
v(t+2) \quad=\sqrt{\frac{1+\gamma(t+1)}{1+\alpha}} v(t+1)+ \\
\sum_{j=1}^{n} \varphi_{j}(t+1)\left(G_{j}(t+1)-x(t+1)\right) .
\end{gathered}
$$

When every best position $G_{j}(t)$ of the $j$ th solution is assumed as constant parameter, (31) subtracts into (29).

$$
\begin{gathered}
v(t+2)-\left(\sqrt{\frac{1+\gamma(t)}{1+\alpha}}+1-\sum_{j=1}^{n} \varphi_{j}(t)\right) v(t+1)+ \\
\sqrt{\frac{1+\gamma(t)}{1+\alpha}} v(t)=0 .
\end{gathered}
$$

For simplify, the equation (32) can be also rewritten as

$$
v(t+2)-\zeta(t+1) v(t+1)+\psi(t) v(t)=0
$$

where

$$
\psi(t+1)=\sqrt{\frac{1+\gamma(t+1)}{1+\alpha}}
$$

and

$$
\zeta(t+1)=\psi(t+1)+1-\sum_{j=1}^{n} \varphi_{j}(t+1) .
$$

In essence, the system (33) of ECO algorithm is the time-varying second order linear system with random variables. In order to discuss and analyze the convergence, one discrete Lyapunov function is needed to discuss the stability and the convergence of ECO algorithm.

Assuming $\mathrm{y}(\mathrm{t}+1)=\psi(t) v(t)$, (33) can be also described by

$$
\left\{\begin{array}{c}
v(t+1)=-\zeta(t) v(t)-y(t) \\
y(t+1)=\psi(t) v(t)
\end{array}\right.
$$


From the perspective of the matrix, it can also be rewritten as

$$
M(t+1)=\Gamma(t) M(t)
$$

where

$$
M(t)=\left[\begin{array}{l}
v(t) \\
y(t)
\end{array}\right]
$$

and

$$
\Gamma(t)=\left[\begin{array}{cc}
-\zeta(t) & -1 \\
\psi(t) & 0
\end{array}\right]
$$

\subsubsection{The Simplest Convergence Analysis of ECO Algorithm}

Theorem 1: Consider the constant transfer matrix $\Gamma=\left[\begin{array}{cc}-\zeta & -1 \\ \psi & 0\end{array}\right]$, and time-varying parameters including $\zeta(t)$ and $\psi(t)$ are simply considered to be constant parameters. If and only if those parameters satisfy the following conditions, the solution can be stable and converge into one point.

$$
\left\{\begin{array}{c}
-1<\psi<1 \\
-1-\psi<\zeta<1+\psi
\end{array}\right.
$$

In other words, if and only if the solution converges into one point, those parameters should be yielded to

$$
\left\{\begin{array}{c}
\gamma<\alpha \\
0<\sum_{j=1}^{n} \varphi_{j}<2 \sqrt{\frac{1+\gamma}{1+\alpha}}+2 .
\end{array}\right.
$$

Proof In order to ensure the stability and the convergence of one solution, one discrete Lyapunov function $\mathrm{S}(\mathrm{t})$ should satisfy two following conditions.

$$
\left\{\begin{array}{c}
\forall t S(t)>0 \\
S(t+1)-S(t)<0 .
\end{array}\right.
$$

Consider the discrete Lyapunov function as

$$
S(t)=\frac{1}{\eta} M(t)^{\mathrm{T}} P M(t)
$$

where

$$
\eta=(\psi-1)(\zeta+1+\psi)(\zeta-1-\psi)
$$

and

$$
P=\left[\begin{array}{cc}
\psi^{3}+\psi^{2}+\psi+1 & \left(1+\psi^{2}\right) \zeta \\
\left(1+\psi^{2}\right) \zeta & 2 \psi+2+\psi \zeta^{2}-\zeta^{2}
\end{array}\right] .
$$

Firstly, it needs to prove that the symmetric matrix $\frac{1}{\eta} P$ is the positive definitive matrix, therefore, the parameters including $\zeta$ and $\psi$ should satisfy the following conditions.

1. The first condition can be mathematically described by

$$
\eta=(\psi-1)\left(\zeta^{2}-(1+\psi)^{2}\right)>0 .
$$

2. The second condition can be mathematically expressed by

$$
\frac{\psi^{3}+\psi^{2}+\psi+1}{\eta}=\frac{\psi^{3}+\psi^{2}+\psi+1}{(\psi-1)\left(\zeta^{2}-(1+\psi)^{2}\right)}>0 .
$$

3. Finally, the third condition can be mathematically written by

$$
\frac{1}{\eta^{2}}\left|\begin{array}{cc}
\psi^{3}+\psi^{2}+\psi+1 & \left(1+\psi^{2}\right) \zeta \\
\left(1+\psi^{2}\right) \zeta & 2 \psi+2+\psi \zeta^{2}-\zeta^{2}
\end{array}\right|>0 .
$$

In other words, the equation (48) can also be expressed by

$$
\begin{gathered}
\frac{1}{\eta^{2}}\left[\left(\psi^{3}+\psi^{2}+\psi+1\right) \times\left(2 \psi+2+\psi \zeta^{2}-\zeta^{2}\right)-\right. \\
\left.\left(1+\psi^{2}\right)^{2} \zeta^{2}\right]>0 .
\end{gathered}
$$

For simplify, the equation (48) can also be rewritten as

$$
\frac{1}{\eta^{2}} \times 2\left(1+\psi^{2}\right)(\psi+1+\zeta)(\psi+1-\zeta)>0 .
$$

As a result, the equation (50) can imply that

$$
-\psi-1<\zeta<\psi+1
$$

and

$$
\psi>\zeta-1 .
$$

Inserting (52) into (46), we can obtain

$$
\psi<1 .
$$


Also inserting (52) into (47), we can get

$$
-1<\psi<1
$$

According to the condition (41), we can conclude that the matrix $P$ is the positive definitive matrix.

Secondly, we also need to prove that $\Delta S(t)$ is strictly smaller than 0 according to the abovementioned Lyapunov function.

Therefore, $\Delta S(t)=S(t+1)-S(t)$ can be mathematically calculated by

$$
\triangle S(t)=\frac{1}{\eta}\left[M(t+1)^{\mathrm{T}} P M(t+1)-M(t)^{\mathrm{T}} P M(t)\right] .
$$

Hence, $\Delta S(t)$ can also be rewritten as

$$
\triangle S(t)=\frac{1}{\eta} M(t)^{\mathrm{T}}\left(\Gamma^{\mathrm{T}} P \Gamma-P\right) M(t) .
$$

Specifically speaking, it is natural to calculate $\Gamma^{\mathrm{T}} P \Gamma$ that

$$
\Gamma^{\mathrm{T}} P \Gamma=\left[\begin{array}{cc}
2 \psi^{3}+2 \psi^{2}-\psi \zeta^{2}+\zeta^{2} & \zeta+\zeta \psi^{2} \\
\zeta+\zeta \psi^{2} & \psi^{3}+\psi^{2}+\psi+1
\end{array}\right] .
$$

Then, the equation of $\Gamma^{\mathrm{T}} P \Gamma-P$ is

$$
\Gamma^{\mathrm{T}} P \Gamma-P=\left[\begin{array}{cc}
-\eta & 0 \\
0 & -\eta
\end{array}\right]
$$

Finally, the equation $\Delta S(t)$ can be calculated by

$$
\Delta S(t)=\frac{1}{\eta} M(t)^{\mathrm{T}}\left(\Gamma^{\mathrm{T}} P \Gamma-P\right) M(t)=M(t)^{\mathrm{T}}\left[\begin{array}{cc}
-1 & 0 \\
0 & -1
\end{array}\right] M(t) \leqslant 0 .
$$

\subsubsection{One Random Convergence Analysis of ECO Algorithm}

Theorem 2: Consider one time-varying ECO system, and its transfer matrix $\Gamma_{1}(t)$ is equal to $\left[\begin{array}{cc}-\zeta(t) & -1 \\ \psi & 0\end{array}\right]$, where $\psi(t)$ is considered to be the constant parameter. If and only if one solution should be converge into one point, those parameters should be yielded to

$$
\left\{\begin{array}{c}
-1<\psi<1 \\
-1-\psi<\zeta<1+\psi \\
(\psi-1)^{2}\left[\zeta(t+1)^{2}-(1+\psi)^{2}\right]\left[\zeta(t)^{2}-(1+\psi)^{2}\right] \\
<\left(1+\psi^{2}\right)^{2}[\zeta(t+1)-\zeta(t)]^{2}
\end{array}\right.
$$

In other words, those parameters, including $\gamma, \alpha$ and $\sum_{j=1}^{n} \varphi_{j}$, should satisfy

$$
\left\{\begin{array}{c}
\gamma<\alpha \\
0<\sum_{j=1}^{n} \varphi_{j}<2 \sqrt{\frac{1+\gamma}{1+\alpha}}+2 \\
\left(\sqrt{\frac{1+\gamma}{1+\alpha}}-1\right)^{2}\left[2 \sqrt{\frac{1+\gamma}{1+\alpha}}+2-\sum_{j=1}^{n} \varphi_{j}(t+1)\right]\left[2 \sqrt{\frac{1+\gamma}{1+\alpha}}+2\right. \\
\left.-\sum_{j=1}^{n} \varphi_{j}(t)\right]>\frac{(2+\gamma+\alpha)^{2}\left[\sum_{j=1}^{n} \varphi_{j}(t+1)-\sum_{j=1}^{n} \varphi_{j}(t)\right]}{(1+\alpha)^{2} \sum_{j=1}^{n} \varphi_{j}(t+1) \sum_{j=1}^{n} \varphi_{j}(t)} .
\end{array}\right.
$$

Proof Consider one discrete Lyapunov function as

$$
S_{1}(t)=M(t)^{\mathrm{T}} P_{1}(t) M(t)
$$

where the symmetric matrix $P_{1}(t)$ is

$$
P_{1}(t)=\left[\begin{array}{cc}
\psi^{3}+\psi^{2}+\psi+1 & \left(1+\psi^{2}\right) \zeta(t) \\
\left(1+\psi^{2}\right) \zeta(t) & 2 \psi+2+\psi \zeta(t)^{2}-\zeta(t)^{2}
\end{array}\right] .
$$

On one hand, by using the above-mentioned method, we need to prove that the symmetric $P(t)$ is the positive matrix. In order to achieve this task, there are two typical conditions to be satisfied

1. The first leading minor of symmetric matrix $P_{1}(t)$ is strictly larger than 0 .

$$
\psi^{3}+\psi^{2}+\psi+1>0
$$

According to the equation (64), we can obtain

$$
\psi>-1
$$

2. The second principal minor of symmetric matrix $P_{1}(t)$ is also strictly larger than 0 .

$$
\left|\begin{array}{cc}
\psi^{3}+\psi^{2}+\psi+1 & \left(1+\psi^{2}\right) \zeta(t) \\
\left(1+\psi^{2}\right) \zeta(t) & 2 \psi+2+\psi \zeta(t)^{2}-\zeta(t)^{2}
\end{array}\right|>0 .
$$


In terms of (64) and (66), we also obtain

$$
\left\{\begin{array}{c}
-1<\psi \\
-1-\psi<\zeta(t)<1+\psi
\end{array}\right.
$$

On the other hand, it needs to prove that $\triangle S_{1}(t)=$ $S_{1}(t+1)-S_{1}(t)$, without $\eta(t)>0$, is a negative definite matrix.

$\Delta S_{1}(t)=M(t+1)^{\mathrm{T}} P_{1}(t+1) M(t+1)-M(t)^{\mathrm{T}} P_{1}(t) M(t)$.

For simplify, the equation (68) can be rewritten as

$\triangle S_{1}(t)=M(t)^{\mathrm{T}}\left[\Gamma(t+1)^{\mathrm{T}} P_{1}(t+1) \Gamma(t+1)-P_{1}(t)\right] M(t)$.

Assuming $Z 1(t)=\Gamma(t+1)^{\mathrm{T}} P_{1}(t+1) \Gamma(t+1)-$ $P_{1}(t)$, and the matrix $\Delta S_{1}(t)$ can be described by

$$
\triangle S_{1}(t)=M(t)^{\mathrm{T}} Z 1(t) M(t)
$$

where

$$
\left\{\begin{array}{c}
Z 1_{11}(t)=-(\psi-1)\left[\zeta(t+1)^{2}-(\psi+1)^{2}\right] \\
Z 1_{12}(t)=-\left(\psi^{2}+1\right)[\zeta(t+1)-\zeta(t)] \\
Z 1_{21}(t)=-\left(\psi^{2}+1\right)[\zeta(t+1)-\zeta(t)] \\
Z 1_{22}(t)=-(\psi-1)\left[\zeta(t)^{2}-(\psi+1)^{2}\right]
\end{array}\right.
$$

In order to establish stability results, there are two conditions which are closely related to leading minor of the first and second order of matrix $Z 1(t)$.

$$
\left\{\begin{array}{c}
Z 1_{11}(t)<0 \\
\operatorname{det}(Z 1(t))=\left|\begin{array}{ll}
Z 1_{11}(t) & Z 1_{12}(t) \\
Z 1_{21}(t) & Z 1_{22}(t)
\end{array}\right|>0
\end{array}\right.
$$

where $\operatorname{det}()$ is the operator of getting the determinant value.

To satisfy the equation (72), those parameters should satisfy the following conditions.

$$
\left\{\begin{array}{c}
\psi<1 \\
(\psi-1)^{2}\left[\zeta(t+1)^{2}-(1+\psi)^{2}\right)\left(\zeta(t)^{2}-(1+\psi)^{2}\right] \\
<\left(\psi^{2}+1\right)^{2}[\zeta(t+1)-\zeta(t)]^{2}
\end{array}\right.
$$

Remark 1: According to (67) and (73), those conditions, considering the parameter $\psi$ as the constant value, are equivalent to the necessary and sufficient condition (61) of ECO algorithm.

\subsubsection{Another Random Convergence Analysis of ECO Algorithm}

Theorem 3: Consider another time-varying ECO system, and the corresponding transfer matrix $\gamma(t)$ is $\left[\begin{array}{cc}-\zeta(t) & -1 \\ \psi(t) & 0\end{array}\right]$. If and only if the solution converges into one point, those parameters should satisfy the following conditions.

$$
\left\{\begin{array}{c}
\psi(t)>-1 \\
-1-\psi(t)<\zeta(t)<1+\psi(t) \\
\left|\begin{array}{cc}
Z 2_{11}(t) \mid<0 \\
Z 2_{11}(t) & Z 2_{12}(t) \\
Z 2_{21}(t) & Z 2_{22}(t)
\end{array}\right|>0
\end{array}\right.
$$

where

$$
\left\{\begin{aligned}
Z 2_{11}(t) \quad & 2 \sum_{j=1}^{n} \varphi_{j}(t+1) \psi^{2}(t+1)-2 \sum_{j=1}^{n} \varphi_{j}(t+1)+ \\
& \left(\sum_{j=1}^{n} \varphi_{j}(t+1)\right)^{2}-\psi(t+1)\left(\sum_{j=1}^{n} \varphi_{j}(t+1)\right)^{2}+\varsigma(t) \\
Z 2_{12}(t)= & -\sum_{j=1}^{n} \varphi_{j}(t+1) \psi^{2}(t+1)-\sum_{j=1}^{n} \varphi_{j}(t+1)+\sum_{j=1}^{n} \varphi_{j}(t) \\
& +\psi^{2}(t) \sum_{j=1}^{n} \varphi_{j}(t)+\varsigma(t)
\end{aligned}\right.
$$

Proof Consider another discrete Lyapunov function

$$
S_{2}(t)=M(t)^{\mathrm{T}} P_{2}(t) M(t)
$$

where the symmetric matrix $P_{2}(t)$ is

$$
P_{2}(t)=\left[\begin{array}{cc}
\psi^{3}(t)+\psi^{2}(t)+\psi(t)+1 & \left(1+\psi^{2}(t)\right) \zeta(t) \\
\left(1+\psi^{2}(t)\right) \zeta(t) & 2 \psi(t)+2+\psi(t) \zeta(t)^{2}-\zeta(t)^{2}
\end{array}\right]
$$

According to the first and second conditions in (74), we can infer that 
1. The first leading minor of symmetric matrix $P_{2}(t)$ is strictly larger than 0 .

$$
\psi^{3}(t)+\psi^{2}(t)+\psi(t)+1>0
$$

2. The second principal minor of symmetric matrix $P_{2}(t)$ is also strictly larger than 0 .

$$
\left|\begin{array}{cc}
\psi^{3}(t)+\psi^{2}(t)+\psi(t)+1 & \left(1+\psi^{2}(t)\right) \zeta(t) \\
\left(1+\psi^{2}(t)\right) \zeta(t) & 2 \psi(t)+2+\psi(t) \zeta(t)^{2}-\zeta(t)^{2}
\end{array}\right|
$$

Then, we need to prove that the equation of $\triangle S_{2}(t)=S_{2}(t+1)-S_{2}(t)$ is a negative semi-definite matrix, and it can be described by

$$
\Delta S_{2}(t)=M(t)^{\mathrm{T}}\left[\Gamma(t+1)^{\mathrm{T}} P_{2}(t+1) \Gamma(t+1)-P_{2}(t)\right] M(t) .
$$

Assuming that $Z 2(t)=\Gamma(t+1)^{\mathrm{T}} P_{2}(t+1) \Gamma(t+1)-$ $P_{2}(t)$, and each element in $Z 2(t)$ is

$$
\left\{\begin{array}{c}
Z 2_{11}(t) \quad=\psi^{3}(t+1)-\psi^{3}(t)+\psi^{2}(t+1)-\psi^{2}(t)+ \\
2 \sum_{j=1}^{n} \varphi_{j}(t+1) \psi^{2}(t+1)+\psi(t+1)-2 \sum_{j=1}^{n} \varphi_{j}(t+1)+t \\
\left(\sum_{j=1}^{n} \varphi_{j}(t+1)\right)^{2}-\psi(t+1)\left(\sum_{j=1}^{n} \varphi_{j}(t+1)\right)^{2}-\psi(t) \\
=\psi^{3}(t+1)-\psi^{3}(t)+\psi^{2}(t+1)-\psi^{2}(t)- \\
Z 2_{12}(t) \quad \sum_{j=1}^{n} \varphi_{j}(t+1) \psi^{2}(t+1)+\psi(t+1)-\sum_{j=1}^{n} \varphi_{j}(t+1) \\
-\psi(t)+\sum_{j=1}^{n} \varphi_{j}(t)+\psi^{2}(t) \sum_{j=1}^{n} \varphi_{j}(t) \\
=\psi^{3}(t+1)-\psi^{3}(t)+\psi^{2}(t+1)-\psi^{2}(t)- \\
\sum_{j=1}^{n} \varphi_{j 1}(t+1) \psi^{2}(t+1)+\psi(t+1)-\sum_{j=1}^{n} \varphi_{j}(t+1) \\
-\psi(t)+\sum_{j=1}^{n} \varphi_{j}(t)+\psi^{2}(t) \sum_{j=1}^{n} \varphi_{j}(t) \\
=\psi^{3}(t+1)-\psi^{3}(t)+\psi^{2}(t+1)-\psi^{2}(t)+ \\
\psi(t+1)-\psi(t)+2 \psi^{2}(t) \sum_{j=1}^{n} \varphi_{j}(t)-\psi(t)\left(\sum_{j=1}^{n} \varphi_{j}(t)\right)^{2 t} \\
-2 \sum_{j=1}^{n} \varphi_{j}(t)+\left(\sum_{j=1}^{n} \varphi_{j}(t)\right)^{2} .
\end{array}\right.
$$

In order to simplify those equations including $Z 2_{11}(t), Z 2_{12}(t), Z 2_{21}(t)$ and $Z 2_{22}(t)$, we assume that

$$
\begin{aligned}
\varsigma(t) & =[\psi(t+1)-\psi(t)]\left[\psi^{2}(t+1)+\psi^{2}(t)\right. \\
& +\psi(t+1) \psi(t)+\psi(t+1)+\psi(t)+1] .
\end{aligned}
$$

According to the above-mentioned remarks and the assumption of (82), we can obtain the mathematical expression of $Z 2(t)$, where

$$
\left\{\begin{aligned}
Z 2_{11}(t)= & \varsigma(t)+2 \sum_{j=1}^{n} \varphi_{j}(t+1) \psi^{2}(t+1)-2 \sum_{j=1}^{n} \varphi_{j}(t+1)+ \\
& \left(\sum_{j=1}^{n} \varphi_{j}(t+1)\right)^{2}-\psi(t+1)\left(\sum_{j=1}^{n} \varphi_{j}(t+1)\right)^{2} \\
Z Z Q_{12}(t)= & \varsigma(t)-\sum_{j=1}^{n} \varphi_{j}(t+1) \psi^{2}(t+1)-\sum_{j=1}^{n} \varphi_{j}(t+1) \\
& +\sum_{j=1}^{n} \varphi_{j}(t)+\psi^{2}(t) \sum_{j=1}^{n} \varphi_{j}(t) \\
Z 2_{21}(t)= & \varsigma(t)-\sum_{j=1}^{n} \varphi_{j}(t+1) \psi^{2}(t+1)-\sum_{j=1}^{n} \varphi_{j}(t+1) \\
& +\sum_{j=1}^{n} \varphi_{j}(t)+\psi^{2}(t) \sum_{j=1}^{n} \varphi_{j}(t) \\
= & \varsigma(t)+2 \psi^{2}(t) \sum_{j=1}^{n} \varphi_{j}(t)-\psi(t)\left(\sum_{j=1}^{n} \varphi_{j}(t)\right)^{2} \\
& -2 \sum_{j=1}^{n} \varphi_{j}(t)+\left(\sum_{j=1}^{n} \varphi_{j}(t)\right)^{2} .
\end{aligned}\right.
$$

In terms of the third and fourth conditions in (74), the first leading minor of matrix of $\triangle S_{2}(t)$ is strictly smaller than 0 , while the second principal minor of matrix $\Delta S_{2}(t)$ is strictly larger than 0 . Finally the matrix $\Delta S_{2}(t)$ is a negative semidefinite matrix, resulting in the convergence behavior of each solution.

In summary, the ECO algorithm is one of the adaptive swarm optimization methods. The solution in ECO algorithm avoids the local optimum by increasing the velocity from the potential energy to the power energy. Meanwhile, the solutions can converge into the suboptimal optimum by the friction power. Therefore, the ECO algorithm not only escapes from the local optimum in the early search stage, but also ensures the convergence of all solu2tions.

\section{Optimal Coverage Tunnel Problem by ECO Algorithm}

In order to tackle with the optimal coverage tunnel problem, the objective of this section is to introduce the ECO algorithm to search for the optimal position of each sensor, fully covering the whole tunnel. 
Step 1: Initialize the parameters of the ECO algorithm and the optimal coverage tunnel problem. Firstly, we set the dimension of solution space on the basis of the number of sensors, the number of all solutions, the topology $E$ of all solutions, the previous best positions $G$ of each solution, the damping factor $\alpha$, the interchanged factor $\beta$, etc.

Step 2: Calculate the fitness of the $i$ th solution according to (6) for the optimal line tunnel coverage problem or (8) for the optimal complicated tunnel coverage problem, the corresponding best position $G_{i}(t)$ is updated according to (84).

$$
G_{i}(t+1)= \begin{cases}X_{i}(t) & \text { if } f\left(X_{i}(t)\right)<f\left(G_{i}(t)\right) \\ G_{i}(t) & \text { if } f\left(X_{i}(t)\right) \geqslant f\left(G_{i}(t)\right)\end{cases}
$$

where $t$ is the current number of iterations and the function $f(\cdot)$ denotes the objective function, respectively.

Step 3: Calculate the varying height $H_{i}(t)$ of each solution according to (6) and (8), and $H_{i}(t)$ can be calculated by

$$
H_{i}(t)=f\left(G_{i}(t)\right)-f\left(G_{i}(t-1)\right)
$$

and

$$
\gamma_{(}(t)=\frac{2 \beta g H_{i}(t)}{v(t)} .
$$

Step 4: In the ECO algorithm, the velocity and the position of each solution can be mathematically described by

$$
\begin{gathered}
v(t+1)=\sqrt{\frac{1+\gamma(t)}{1+\alpha}} v(t)+\sum_{k=1}^{n} c_{k} r_{k} e_{k}\left(G_{k}(t)-x(t)\right) \\
x(t+1)=v(t+1)+x(t)
\end{gathered}
$$

Step 5: Determine whether the current position and the current velocity are beyond the corresponding minimum and maximum or not. The current value is set by the following equations.

$$
\begin{aligned}
& x(t)=x_{\text {min }}+0.2 r_{1}\left(x_{\max }-x_{\min }\right) \quad x(t)<x_{\text {min }} \\
& x(t)=x_{\text {min }}+\left(0.2 r_{2}+0.8\right)\left(x_{\max }-x_{\min }\right) \quad x(t)>x_{\max } \\
& v(t)=v_{\min }+0.2 r_{3}\left(v_{\max }-v_{\min }\right) \quad v(t)<v_{\min }
\end{aligned}
$$

$$
v(t)=v_{\min }+\left(0.2 r_{4}+0.8\right)\left(v_{\max }-v_{\min }\right) \quad v(t)>v_{\max }
$$

where the random variables, including $r_{1}, r_{2}, r_{3}$ and $r_{4}$, are changing in the range from 0 to 1 .

Step 6: Testify whether the stopping condition is satisfied or not. If the stopping condition is satisfied, stop this algorithm; otherwise, go to Step 2.

There are several main results and remarks on energy conservation optimization as follows.

1. To effectively avoid premature convergence in the hypersurface landscape, the next position of each solution and the attractor of ECO algorithm are dependent of several previous best positions of its neighbors.

2. This proposed algorithm is essentially inspired by the law of energy conversation, which describes the changed process from potential energy closely relating to objective fitness to power energy.

3. In the ECO algorithm, there are two crucial parameters so-called the damping factor $\alpha$ and the interchanged factor $\beta$. The damping factor $\alpha$ mainly determines the convergence speed of all solutions, while the interchanged factor $\beta$ sets the relationship between power energy and potential energy.

\section{Numerical Simulations}

To show the feasibility and the performance of ECO algorithm, the objective of this section is to investigate the convergence speed and compare the final optimization result under different parameters and different benchmark functions. Furthermore, those results may provide the guideline for parameter selection on the damping factor $\alpha$ and the corresponding topology $E$, etc.

\subsection{Benchmark Functions}

To demonstrate the effectiveness of ECO algorithm, this subsection is to introduce several typical benchmark functions which are composed of Sphere function, Griewank function, Rastrigin function, Rosenbrock function and Schaffer's f6 function, and so on. 
Sphere function has no local optimums in the hypersurface landscape and denotes the ideal optimization problem. In order to increase the complexity of Sphere function, the modified Sphere function can be mathematically expressed by

$$
f_{1}(\mathbf{x})=\sum_{i=1}^{D}\left(x_{i}+50\right)^{2}
$$

where $D$ denotes the dimension of the whole solution space.

Griewank function, which possibly denotes the practical optimization problem and has a large number of local optimums in the hypersurface landscape, can be mathematically described by

$$
f_{2}(\mathbf{x})=\frac{1}{4000} \sum_{i=1}^{D} x_{i}^{2}-\prod_{i=1}^{N} \cos \left(\frac{x_{i}}{\sqrt{i}}\right)+1 .
$$

In the case of Rastrigin function, it is hard to search for the suboptimal or global optimum in this hypersurface landscape. Rastrigin function, which also has many local optimums, can be mathematically expressed by

$$
f_{3}(\mathbf{x})=\sum_{i=1}^{D}\left(x_{i}-10 \cos \left(2 \pi x_{i}\right)+10\right) .
$$

Rosenbrock function has only few local optimums in the solution space, and the region around the global optimum is very flat. More specifically, Rosenbrock function can be mathematically described by

$$
f_{4}(\mathbf{x})=\sum_{i=1}^{D-1}\left(100\left(x_{i+1}-x_{i}^{2}\right)^{2}+\left(x_{i}-1\right)^{2}\right) .
$$

Schaffer's f6 function also has many local optimums around the global optimum. And all solutions easily get into the local optimum and never get away from many local optimums, finally resulting in premature convergence. Schaffer's f6 function can be mathematically expressed by

$$
f_{5}(x, y)=0.5+\frac{\left(\sin \sqrt{x^{2}+y^{2}}\right)-0.5}{\left(1+0.001\left(x^{2}+y^{2}\right)\right)^{2}} .
$$

Generally speaking, many benchmark functions have the initial ranges on the position and the velocity of all solutions, and those initial ranges are listed in Tab. 1.

\subsection{The Detailed Evolutionary Process of ECO Algorithm}

According to the law of energy conservation, each solution has its own power energy, which is closely related to the velocity, and potential energy is closely related to the changed objective fitness in the hypersurface landscape. The main idea of ECO algorithm is to change potential energy into power energy during the evolutionary process. In other words, when one solution searches for good solution, its potential energy should be decreased right now and the corresponding velocity also increases to avoid premature convergence.

To better illustrate the evolutionary process of energy conversation optimization, the objective of this subsection is to investigate the detailed evolutionary process which mainly involves in swarm topology, convergence speed of all solutions, the corresponding power energy and objective fitness in the whole evolutionary process, etc.

Schaffer's f6 function, which has many local optimums in the hypersurface solution space, is selected to be the considered objective function. The dimension of solution space is set to 2 and the number of all solutions is 10 , respectively. Concerning the damping factor $\alpha$, the parameter $\alpha$ linearly increases from 0.00 to 0.03 to ensure exploration ability at the beginning of search stage and exploitation ability at the end of search stage. The maximum number of generations is set to 1000 and the topology is randomly based on adjacency matrix which 
can be mathematically expressed by

$$
E=\left[\begin{array}{llllllllll}
1 & 1 & 0 & 1 & 0 & 1 & 0 & 0 & 1 & 0 \\
1 & 1 & 1 & 1 & 1 & 0 & 0 & 1 & 0 & 1 \\
1 & 0 & 1 & 1 & 0 & 1 & 1 & 0 & 1 & 1 \\
0 & 0 & 1 & 1 & 1 & 0 & 0 & 0 & 1 & 0 \\
0 & 0 & 1 & 1 & 1 & 0 & 0 & 0 & 0 & 0 \\
0 & 1 & 0 & 1 & 0 & 1 & 1 & 0 & 0 & 0 \\
1 & 1 & 1 & 0 & 1 & 0 & 1 & 1 & 0 & 0 \\
1 & 1 & 1 & 0 & 0 & 1 & 0 & 1 & 0 & 1 \\
0 & 1 & 1 & 0 & 0 & 0 & 0 & 1 & 1 & 1 \\
0 & 1 & 1 & 0 & 0 & 0 & 1 & 1 & 1 & 1
\end{array}\right]
$$

According to adjacency matrix $E$, Laplacian matrix $L$ can be calculated as

$$
L=\left[\begin{array}{cccccccccc}
4 & -1 & 0 & -1 & 0 & -1 & 0 & 0 & -1 & 0 \\
-1 & 6 & -1 & -1 & -1 & 0 & 0 & -1 & 0 & -1 \\
-1 & 0 & 6 & -1 & 0 & -1 & -1 & 0 & -1 & -1 \\
0 & 0 & -1 & 3 & -1 & 0 & 0 & 0 & -1 & 0 \\
0 & 0 & -1 & -1 & 2 & 0 & 0 & 0 & 0 & 0 \\
0 & -1 & 0 & -1 & 0 & 3 & -1 & 0 & 0 & 0 \\
-1 & -1 & -1 & 0 & -1 & 0 & 5 & -1 & 0 & 0 \\
-1 & -1 & -1 & 0 & 0 & -1 & 0 & 5 & 0 & -1 \\
0 & -1 & -1 & 0 & 0 & 0 & 0 & -1 & 4 & -1 \\
0 & -1 & -1 & 0 & 0 & 0 & -1 & -1 & -1 & 5
\end{array}\right]
$$

The first smallest eigenvalue $\lambda_{1}$ of Laplacian matrix is equal to 0 and the second smallest eigenvalue $\lambda_{2}$ is 2.1725 . Since the second smallest eigenvalue $\lambda_{2}$ is strictly larger than 0 , the graph of this topology is a connected graph, which gives rise to convergence behavior of all solutions.

According to the law of energy conservation, each particle has its own power energy, which is closely related to the velocity of every particle, and potential energy is closely related to objective fitness in the hypersurface. The main idea of ECO algorithm is to change potential energy into power energy during the evolutionary process, that is to say, when one solution searches for better position, its potential energy should be decreased right now and the corresponding velocity should be increased to avoid premature convergence. To set the relationship between power energy and potential energy under different benchmark functions, the interchanged factor $\beta$ is calculated by the setting maximum velocity and the initial objective fitness. On the basis of (20), the interchanged factor $\beta$ of every solution is $0.0187,0.0181,0.0162,0.0189,0.0165,0.0256$, $0.0203,0.0168,0.0316$ and 0.0473 , respectively.

According to the above-mentioned analysis, the sum energy of all solutions, which is composed of power energy and potential energy, in the whole evolutionary process can be depicted in Fig. 4.

It can be concluded from Fig. 4 that the energy during the whole evolutionary process strictly decreases since there exists the resistance force in the second order linear ECO system. The speed of reducing the energy is mainly determined by the damping factor $\alpha$. In the general case, large $\alpha$ is helpful for the objective function of few local optimums in the hypersurface landscape, small $\alpha$ benefits from the objective function with many local optimums to easily avoid getting into the local optimum.

Roughly speaking, the current power energy of all solutions comes from two aspects, which are composed of the previous power energy and the interchanged energy from potential energy. Power energy directly determines the current velocity of each solution. The large power energy obviously gives rise to the large velocity while small power energy leads to small velocity. Additionally, in order to demonstrate the convergence of ECO algorithm, power energy in the evolutionary process is depicted in Fig. 5, and the velocity norm of all solutions, which is closely related to power energy, is also shown in Fig. 6.

According to Fig. 5 and Fig. 6, power energy is closely related to the velocity of all solutions. At the beginning of evolutionary process, when all solutions can find good position, objective fitness quickly decreases and the corresponding power energy increases. On the basis of the law of energy conservation, potential energy, which denotes the changed objective fitness in the optimization problem, turns into power energy of each solution, to provide the large velocity of all solutions. At the end of evolutionary process, the whole energy reduces because of the resistance force in the ECO system, and power energy, together with the velocity of each solution, also reduces to converge into the subopti- 


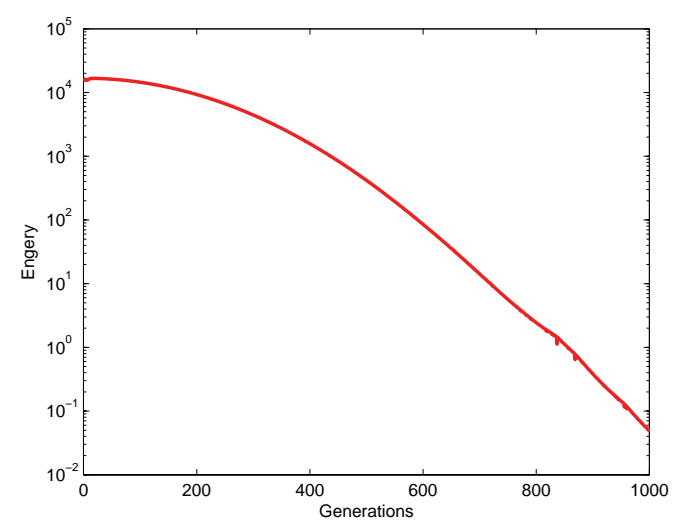

Figure 4: The sum energy of all solutions in the whole process

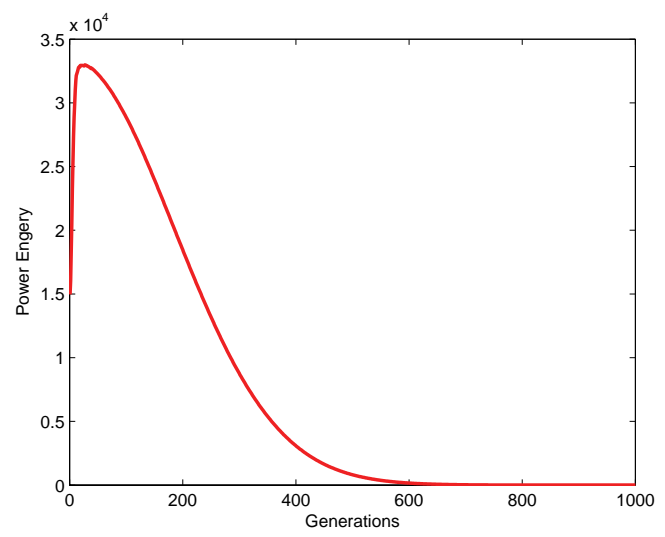

Figure 5: Power energy of all solutions in the evolutionary process

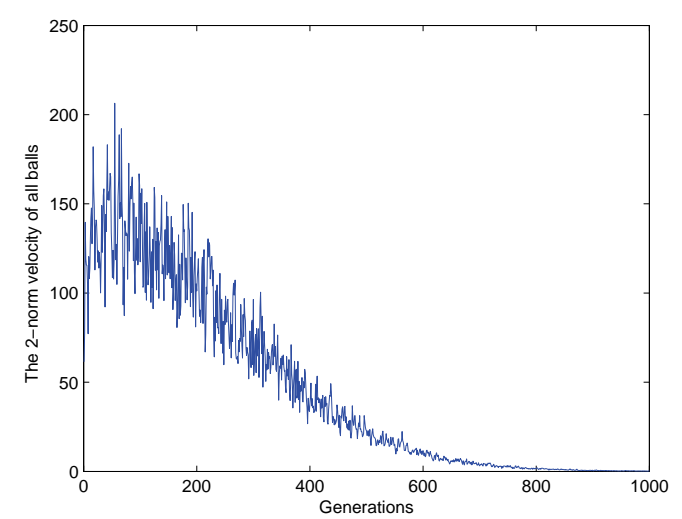

Figure 6: The 2-norm velocity of all solutions in the evolutionary process 
mal or global optimum.

To show the performance of ECO algorithm, the corresponding objective fitness in the whole evolutionary process is depicted in Fig. 7. Totally speaking, the previous objective fitness is larger than that of the current objective fitness. Due to the hypersurface landscape of Schaffer's f6 function, the performance shows that ECO algorithm does not easily get into the local optimum and suffers from premature convergence.

In summary, the above-mentioned discussions mainly focus on the detailed evolutionary process, which mainly consists of swarm topology, the sum energy, power energy and objective function in the whole evolutionary process, etc. In addition, ECO algorithm can balance the tradeoff between exploration ability and exploitation ability by the damping factor $\alpha$ and swarm topology $E$. By adjusting the damping factor $\alpha$ and swarm topology $E$, ECO algorithm can find good solution and effectively avoids premature convergence in the presence of many local optimums in the solution space.

In order to illustrate the key role of the damping factor and swarm topology, energy conversation optimization in the next subsection is applied to find the suboptimal or global optimum under different complicated benchmark functions and different dimensional solution space.

\subsection{Convergence Speed under Different Factors and Topologies}

As is well known, the convergence speed plays a great role on premature convergence, the efficiency of ECO algorithm as well as the tradeoff between exploration ability and exploitation ability, together with the optimization result. In this proposed ECO algorithm, the damping factor $\alpha$ and swarm topology also influences the convergence speed of all solutions. To better illustrate the relationship among them, convergence speed and numerical results are compared under different damping factors and different swarm topologies.

Generally speaking, the damping factor $\alpha$ linearly increases during the whole evolutionary process. The initial damping factor $\alpha$ is set to 0.0 and the final damping factor $\alpha$ linearly increases from
0.01 to 0.05 , respectively. Several power energies under different damping factors are depicted in Fig. 8.

With respect to Fig. 8, the final large damping factor $\alpha$ largely reduces the whole energy and leads to large convergence speed of all solutions. At the beginning of search stage, the power energy quickly increases since potential energy, closely relating to the changed objective function, turns into the power energy to enhance exploration ability of the swarm. The corresponding large velocity possibly is helpful for getting away from the local optimum and effectively avoiding premature convergence. At the end of search stage, the velocity of all solutions is relatively small to find better solution in the adjacent region.

In addition, the objective fitness of each damping factor in the whole evolutionary process is shown in Fig. 9. Due to the complicated landscapes of solution space, all solutions easily suffer from premature convergence. Therefore, at the beginning of evolutionary process, objective fitness quickly reduces and its potential energy turns into power energy to provide large velocity for getting out of local optimum. According to the comparison in Fig. 9, the final damping factor $\alpha$ can be selected to be 0.04 .

Different topologies also play a great role on the convergence speed and the final optimization result. The role of different topologies is to effectively avoid premature convergence since the attractor of each solution is mainly determined by its neighbors with random factor, therefore, all solutions possibly search for the suboptimal or global optimum in many regions. The corresponding power energy and objective fitness during evolutionary process are depicted in Fig. 10 and Fig. 11, respectively. By randomly generated different topologies, the connected link number among all solutions is randomly generated and TP denotes the number of connected links in swarm topology.

In terms of Fig. 10 and Fig. 11, all solutions under different topologies have similar power energy and similar velocity in the evolutionary process. Comparing with different swarm topologies, when the connected link number among all solutions can be selected to 60, ECO algorithm can get 


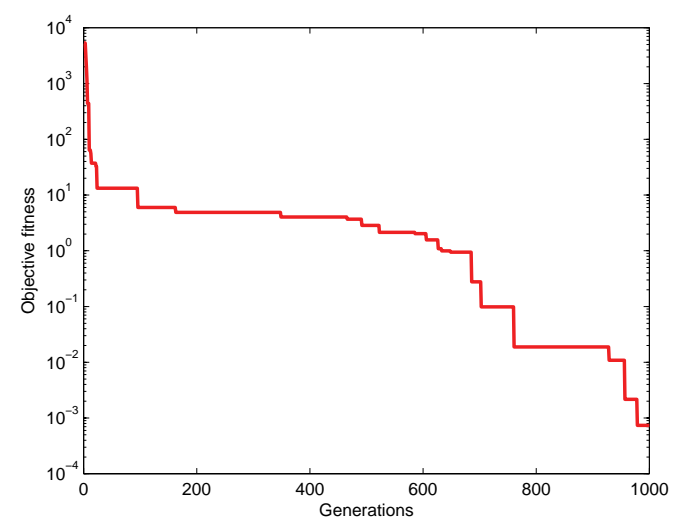

Figure 7: Objective fitness of Schaffer's f6 function in the evolutionary process

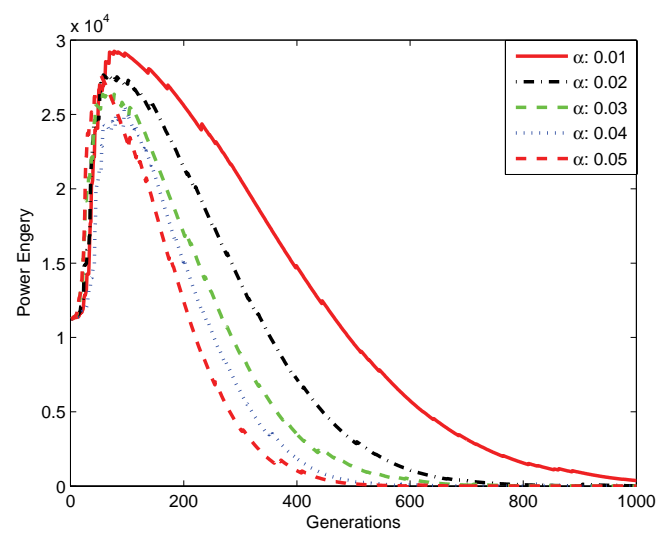

Figure 8: Different damping factors lead to different power energy in the evolutionary process

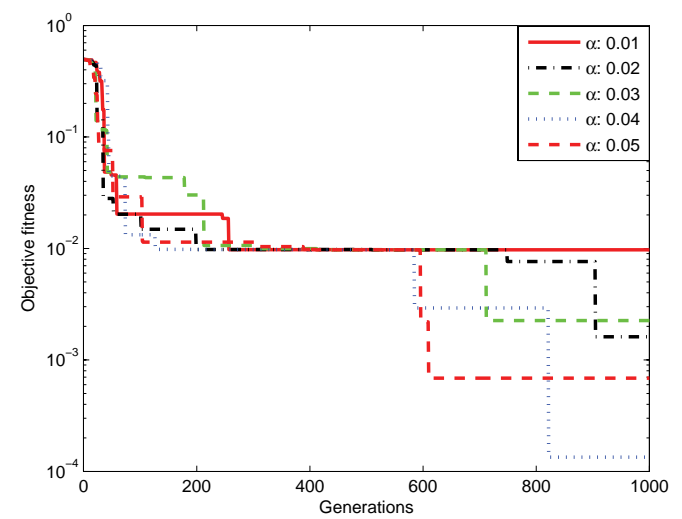

Figure 9: Objective fitness under different damping factors in the evolutionary process 


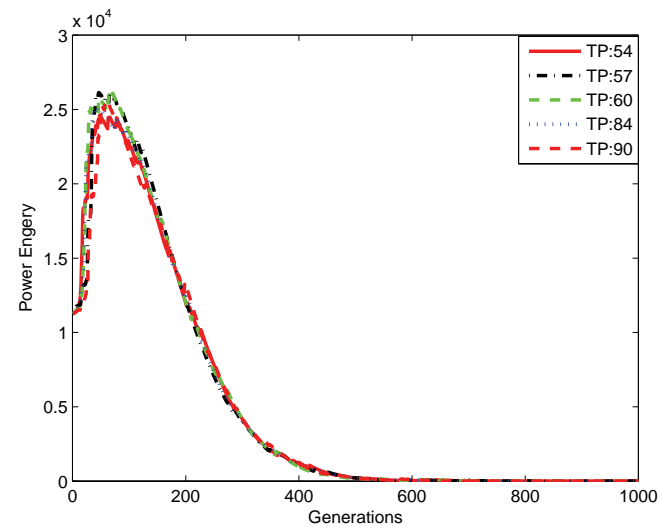

Figure 10: Different swarm topologies lead to different power energy in the evolutionary process

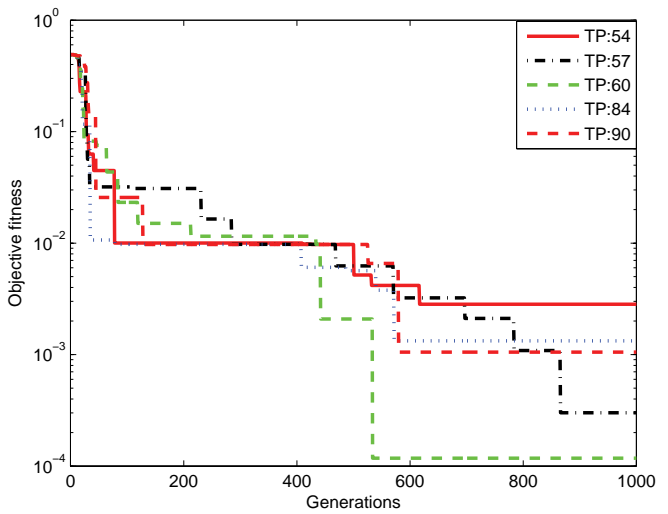

Figure 11: Objective fitness under several different swarm topologies 
good solution. Furthermore, few connected number cannot obtain good solution under Schaffer's f6 function since there is relatively few number of generations in the evolutionary process, and too many connected link number also cannot get good solution since all solutions possibly suffer from premature convergence.

\subsection{Numerical Results under Different Dimensional Spaces}

Concerning the proposed ECO algorithm, one of crucial factors is considered to be the damping factor $\alpha$. At the beginning of search stage, the damping factor $\alpha$ is selected to be small value to enhance exploration ability of all solutions, while the damping factor $\alpha$ at the end of search stage can be selected by large value to improve exploitation ability of all solutions.

In order to investigate final results under different damping factors, corresponding results on the modified Sphere function are concluded in Tab. 2, where every datum is the mean of 50 independent results. According to Tab. 2, large $\alpha$ is favor for finding the suboptimal or global solution in the modified Sphere function. Since large $\alpha$ makes the energy to quickly reduce in the evolutionary process, the velocity of all solutions becomes smaller and smaller, resulting in exploitation ability of the swarm. In addition, the modified Sphere function has no local optimum and all solutions do not suffer from premature convergence, therefore, large $\alpha$ benefits the optimization problem which has few local optimums or without local optimums.

In the case of Griewank function, there are many local optimums in the hypersurface landscape, several results from 2 dimensions to 20 dimensions are listed in Tab. 3. According to Tab. 3, the parameter $\alpha$ from 0 to 0.05 is the optimal parameter, which can lead to good solution in Griewank function.

With respect to Rastrigin function, there are also many local optimums in the complicated solution space, leading to the uncertainty of final results in Tab. 4. In the low dimensional space, the optimal parameter $\alpha$ so-called the damping factor can be selected to $[\mathbf{0 , 0 . 0 2}]$ or $[\mathbf{0 , 0 . 0 3 ]}$. In the high dimensional space, the corresponding optimal parameter can be selected to $[\mathbf{0 , 0 . 0 4 ]}$ or $[\mathbf{0 , 0 . 0 5}$.

Regarding Rosenbrock function, it is also difficult to find the suboptimal or global solution in this hypersurface landscape and the corresponding results are listed in Tab. 5 from 2 dimensions to 20 dimensions. In the low dimensional space, the best parameter $\alpha$ can be selected to $[\mathbf{0 , 0 . 0 4 ]}$ or $[\mathbf{0 ,}$ 0.05], getting better solution. In the high dimensional space, the best parameter $\alpha$ can be selected to $[\mathbf{0 , 0 . 0 2}]$ or $[\mathbf{0 , 0 . 0 3 ]}$, the others possibly result in early getting into the local optimum.

In the 2-dimensional solution space, all solutions easily plunge into many local optimums due to the complicated hypersurface of Schaffer's f6 function. According to Tab. 6, the best parameter $\alpha$ can be selected to $[\mathbf{0 , 0 . 0 5 ]}$ and the corresponding optimization result is equivalent to 0.0020 .

According to the comparison under different $\alpha$, this parameter is better to select $[\mathbf{0 , 0 . 0 4 ]}$ or $[\mathbf{0}$, 0.05], that is to say, the damping factor $\alpha$ increases linearly from 0.0 to 0.05 in the whole evolutionary process.

\subsection{Comparison with Particle Swarm Optimization}

In order to compare with the final optimization results by the standard PSO algorithm, the inertia weight method $[26,15]$ and the constriction factor method [17] in PSO algorithm are selected to compare the advantage and the disadvantage of ECO algorithm. Particle swarm optimization is the typical population-based optimization and can find good solution in the different hypersurfaces. In the inertia weight method, the inertia weight $\omega$ linearly decreases from 0.9 to 0.4 in the evolutionary process, and acceleration coefficients are generally set to 2.0. In the constriction factor method, the constriction factor is selected to be 0.729 and acceleration coefficients are also equivalent to 2.0. In addition, the damping factor $\alpha$ in ECO algorithm linearly increases from 0 to 0.05 in the whole evolutionary process. To demonstrate the advantage and the disadvantage of ECO algorithm, the corresponding results from 3 dimensions to 30 dimensions are compared in Tab. 7, Tab. 8 and Tab. 9 under Griewank function, Rastrigin function and Schaffer's f6 func- 
tion, respectively. In addition, the number of generations during the whole evolutionary process is set to 1000 , while the number of all solutions is set to 30.

According to Tab. 7 under Griewank function, when the dimension of solution space is smaller than 18 , the inertia weight method in PSO algorithm can get the best solution. When the dimension of solution space is larger than 18, energy conversation optimization can get good solution. In addition, the constriction factor method in PSO algorithm is not selected to be better method in the low or high dimensional space.

On the basis of Tab. 8 under Rastrigin function, when the dimension of solution space is smaller than 21 , the best method is selected to be the inertia weight method; when the dimension of solution is larger than 21, ECO algorithm can get the best solution. Furthermore, the constriction factor method does not obtain the good solution due to the large convergence speed and easily getting into the local optimum.

According to Tab. 9 under Schaffer's f6 function, the best result is obtained by energy conversation optimization under Schaffer's f6 function and the corresponding optimization result is equivalent to 0.0024 . Due to the complicated hypersurface of Schaffer's f6 function, energy conversation optimization is possible to obtain good solution under many local optimums to avoid premature convergence.

To better investigate the performance among three typical algorithms, objective finesses under three algorithms are compared in the whole evolutionary process. The corresponding comparison under three typical evolutionary methods is depicted in Fig. 12.

\section{Optimal Coverage Tunnels Problem by ECO Algorithm}

In order to demonstrate the effectiveness of ECO algorithm for the optimal tunnel coverage problem, numerical results mainly concentrate on four different typical tunnels to be fully covered. The main task of ECO algorithm is to search for the optimal position of each sensor, so that the sensor network can fully cover the setting tunnel in the different mountainous scenarios. Since the tunnel in the highspeed railway is typically classified by the line tunnel and the complicated tunnel, therefore, numerical results mainly discuss one case with one line tunnel, another case with several line tunnels, the third case with the complicated tunnel and the fourth case with several complicated tunnels.

\subsection{One Case with One Line Tunnel}

Let the length of the line tunnel and the sensor number in sensor network be 2034 meters and 5, respectively. The covered radius of each sensor is assumed to be 200 meters. The number of solutions in ECO algorithm is set to 20 , while the maximum number of generations is assumed to be 1000 . The parameter $\alpha$ in (27) is set to 0.5. By utilizing the ECO algorithm, the computational time of ECO algorithm is 0.922 seconds. As depicted in Fig. 13, the optimal sensor positions are $205.7 \mathrm{~m}, 611.3 \mathrm{~m}, 1017 \mathrm{~m}$ and $1828.3 \mathrm{~m}$. Notice that the distance between two adjacent sensors is $5.667 \mathrm{~m}$, not fully covering the whole line tunnel.

Assume the length of the line tunnel and the number of sensors be 4030 meters and 10, respectively. Other parameters are equal to those of the above-mentioned case. By the new ECO algorithm, the computational time is 0.969 seconds. Additionally, as shown in Fig. 14, the positions of all sensors are $202.6 \mathrm{~m}, 605.3 \mathrm{~m}, 1007.9 \mathrm{~m}, 1410.6 \mathrm{~m}, 1813.3 \mathrm{~m}$, $2216 \mathrm{~m}, 2618.7 \mathrm{~m}, 3021.5 \mathrm{~m}, 3424.3 \mathrm{~m}$ and $3827.1 \mathrm{~m}$. The overlapping distance between two sensors is 2.727 meters, leading to fully covering the line tunnel.

\subsection{Another Case with Several Line Tunnels}

In the practical mountainous scenarios, the whole length of tunnel is 1647 meters, and the range of tunnels are set to $(0,140),(305,467),(597,738)$, $(892,1026),(1172,1338)$ and $(1498,1647)$. In order to effectively protect the sensor, it is of importance to ensure that the sensors should be set in the tunnel, mainly avoiding the rain. Let the number of sensors in sensor network be 5. By using the 


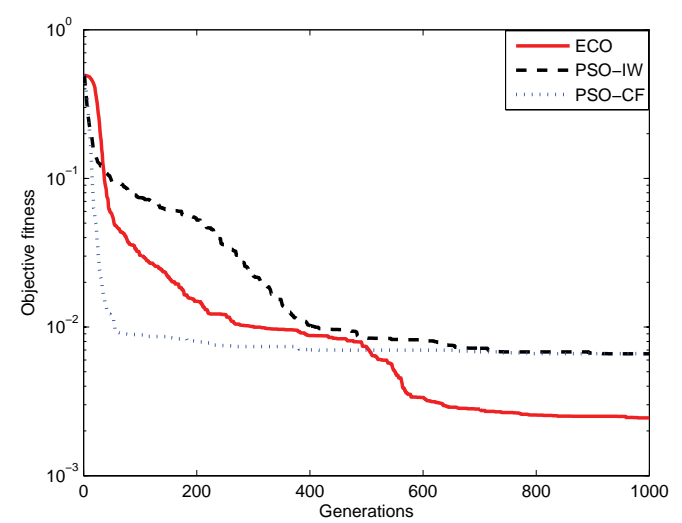

Figure 12: Comparing with two typical methods in the standard PSO algorithm

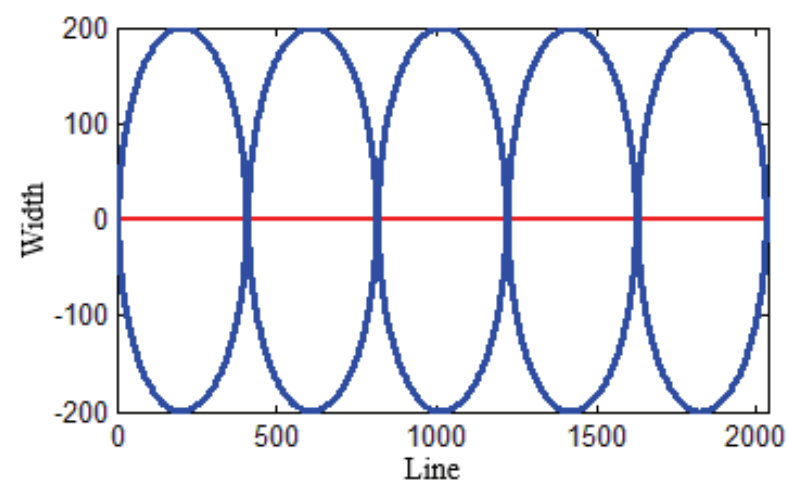

Figure 13: Case 1: The optimal line tunnel $(\mathrm{L}=2034)$

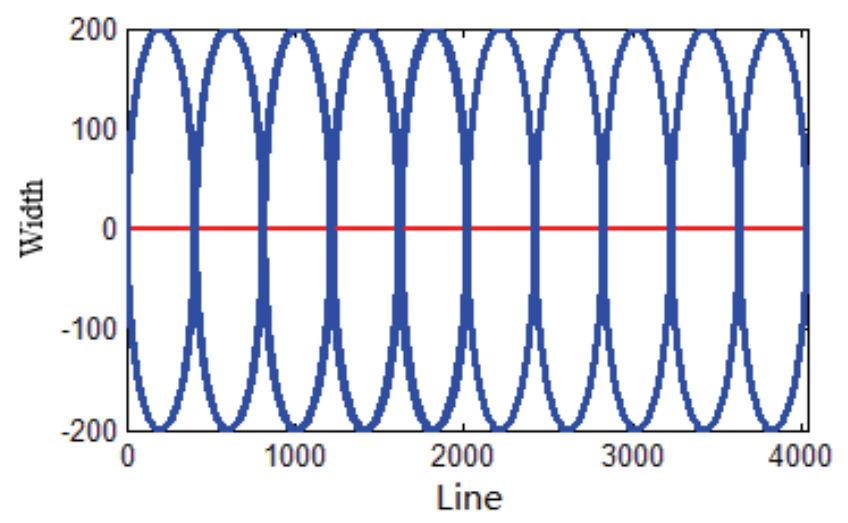

Figure 14: Case 1: The optimal line tunnel $(\mathrm{L}=4030)$ 


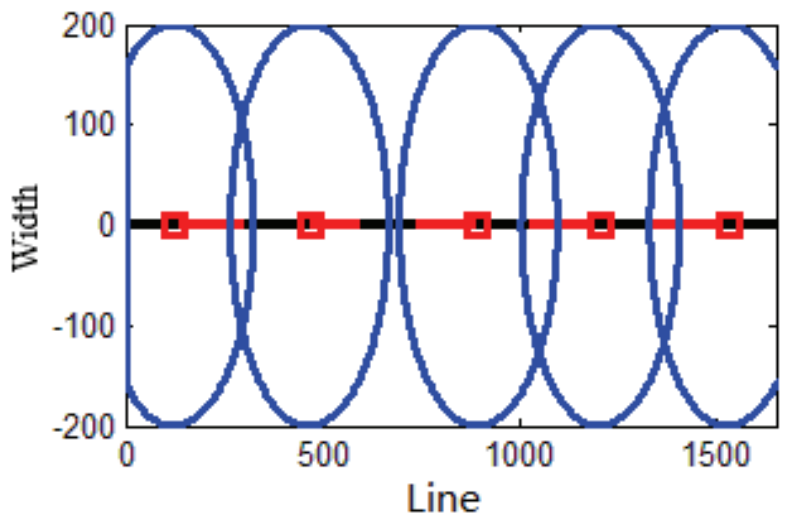

Figure 15: Case 2: The optimal line tunnels by ECO algorithm $(\mathrm{L}=1647)$

ECO algorithm, its computational time is $2.735 \mathrm{sec}-$ onds, and the optimal position of each sensor is set to $124.8 \mathrm{~m}, 466.1 \mathrm{~m}, 893.5 \mathrm{~m}, 1202.2 \mathrm{~m}$ and $1527.2 \mathrm{~m}$. As depicted in Fig. 15, the black line denotes the railway in the tunnel and the red line denotes the railway between two tunnels. Additionally, the red square denotes the optimal position of each sensor, finally leading to fully covering the line tunnels.

\subsection{Third Case with the Complicated Tunnel}

Taking the different complicated tunnels in the mountainous environment into account, it is so difficult to set up the mathematical model of each tunnel, therefore, we highlight that the corresponding tunnel consists of the order discrete points along the railway. In order to deeply study the relationship between the radius of sensor and the optimal coverage tunnel problem, the subsection is to discuss the optimal coverage tunnel problem in the presence of different sensor radii.

There are 200 discrete points along the railway and the sensor radius is set to 20 , and the number of sensors is equal to 5. By the ECO algorithm, the computational time is 34.5 seconds since each evolutionary step should determine whether each discrete point is covered by the sensor network or not, and the sensor network cannot fully cover the tunnel. As depicted in Fig. 16, the optimal positions of those sensors are 20, 58, 104, 144 and 181, respectively.

When the radius of each sensor is equal to 22 , the computational time by the ECO algorithm is 37.75 seconds. As shown in Fig. 17, the corresponding optimal position of each sensor is $22,65,105,148$ and 188 , giving rise to fully covering the whole given tunnel.

When the radius of sensor is set to 24 , the computational time by the ECO algorithm is $34.42 \mathrm{sec}-$ onds, meanwhile, the corresponding position of sensor is 13, 58, 102, 149 and 193. Furthermore, the sensor network can fully cover the whole tunnel and several discrete points in the tunnel are covered by two sensors.

Comparing with the three above-mentioned results, the optimal radius of sensor is set to 22 , and the sensor network fully covers the whole tunnel. Moreover, few discrete points in the tunnel are covered by two adjacency sensors.

\subsection{Fourth Case with Several Complicated Tunnels}

As for the optimal coverage tunnel problem for several complicated tunnels, the radius of sensor is set to 24, and the computational time of the ECO algorithm is 36.07 seconds. The sensor network can fully cover the discrete points in the tunnel. In order to protect the sensors in the tunnel, the sensors should also be in the tunnel. By the ECO algorithm, the optimal position of each sensor, which is roughly shown in Fig. 19, is 3, 50, 97, 139 and 183, respectively. 


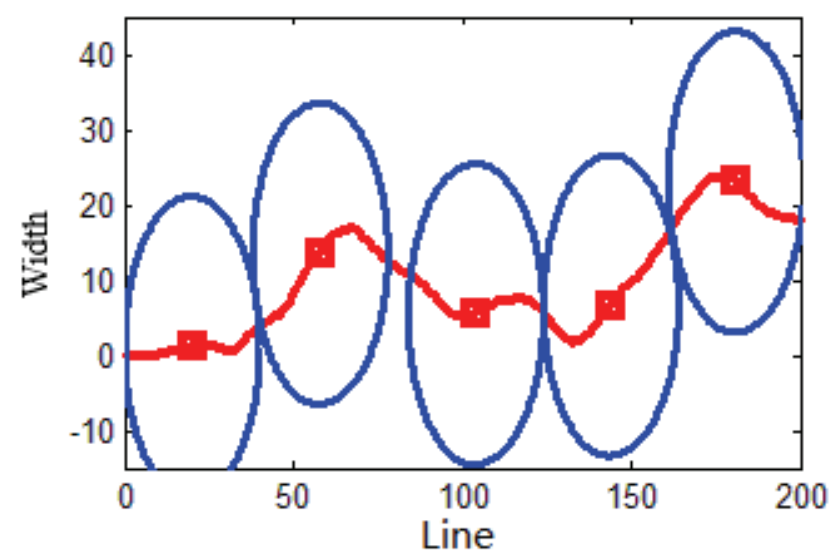

Figure 16: Case 3: The optimal complicated tunnels by ECO algorithm $(\mathrm{R}=20)$

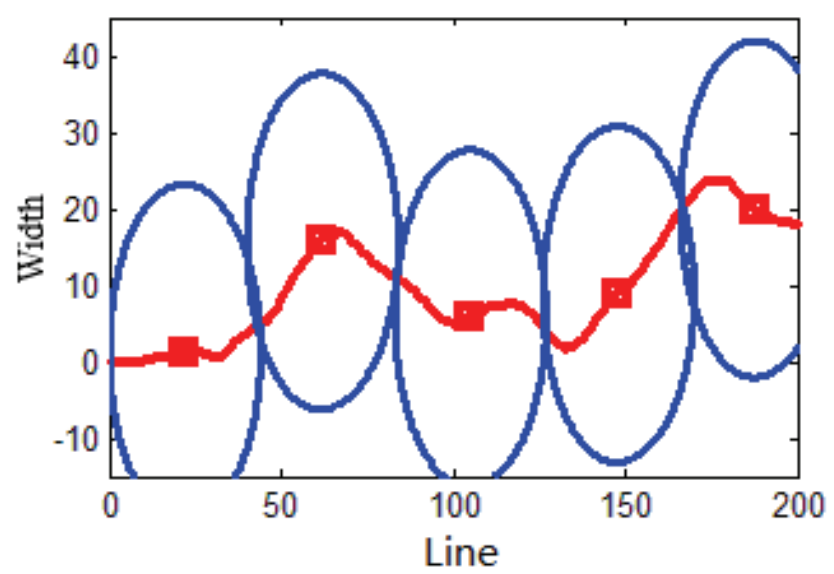

Figure 17: Case 3: The optimal complicated tunnels by ECO algorithm $(\mathrm{R}=22)$

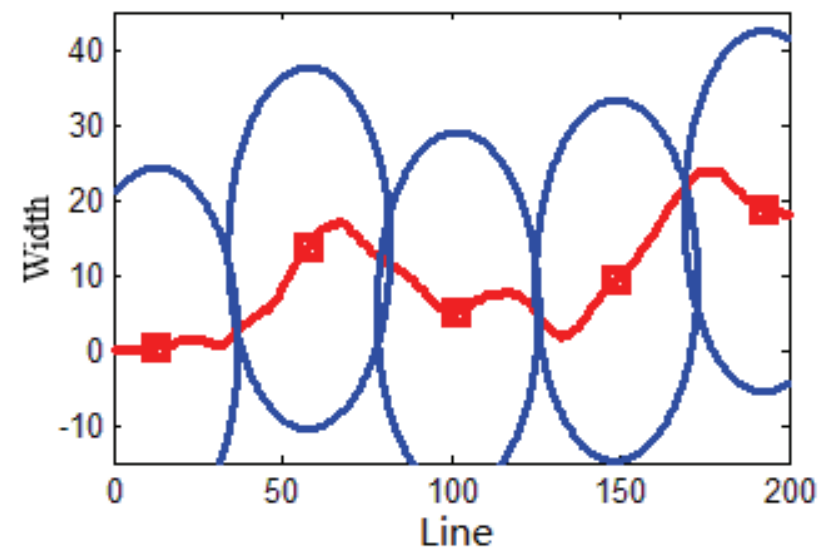

Figure 18: Case 3: The optimal complicated tunnels by ECO algorithm $(\mathrm{R}=22)$ 


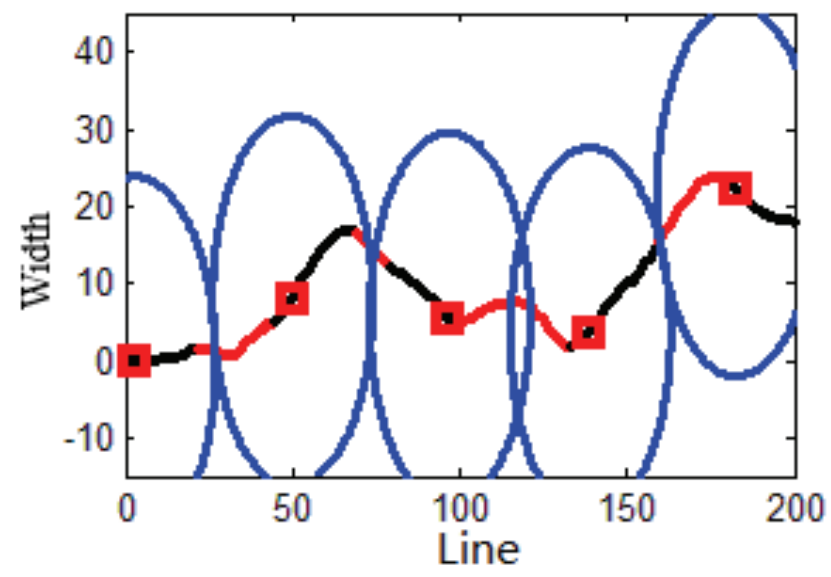

Figure 19: Case 4: The optimal complicated tunnels by ECO algorithm

\section{Conclusion and Future Works}

The optimal tunnel coverage problem is mainly and successfully solved by one new nature-inspired swarm optimization method so-called ECO algorithm. In order to better handle with the optimal tunnel coverage problem, the corresponding objective function and its constraints are given to mathematically analyze the different complicated tunnels and the tunnel coverage problem in the presence of several basic assumptions and concepts. Moreover, the new ECO algorithm, which is chiefly motivated by the law of energy conversation and PSO algorithm, is introduced to show the basic concepts and main steps of ECO algorithm and copy with the optimal tunnel coverage problem. For the sake of demonstrating the effectiveness of ECO algorithm, simulation results mainly focus on the different typical tunnels to be covered, such as the line tunnel, the indirected line tunnel and the complicated tunnel, etc.

In this paper, there are several interesting and unsolved problems in the case of energy conversation optimization.

1. Due to random numbers in ECO algorithm, the brief conditions in the case of convergence analysis may be a challenging problem, which is actually helpful for parameter selection and suitable topology for different optimization problems.
2. To get better solution in the low dimensional space, parameter selection and swarm topology possibly play a great role on the efficiency of ECO algorithm in the future.

3. Energy conversation optimization can be applied to the ranges of neural network, PID control, system identification and power system, other practical applications, etc.

The future work mainly concentrates on the realistic application of tunnel coverage under the different scenarios. It is of importance to develop the tunnel coverage software by ECO algorithm on GIS system to locate the time-varying train in the tunnel or mountain. Additionally, it is necessary to discuss the large number of sensors to cover the given long tunnel by the improved ECO algorithm.

\section{Acknowledgments}

This work is mainly supported by China Postdoctoral Science Foundation (2014M550988). This work is also supported by the China's Railway Corporation under Grants 2013X009-A-1 and 2013X009-A-2. Additionally, this work is supported by National Natural Science Foundation of China (61273150, 61321002, 61433003, 61473038) and Doctoral Program of Higher Education of China (20121101110029). 


\section{References}

[1] S. S. Saab. A map matching approach for train positioning part I development and analysis. IEEE Transactions on Vehicular Technology, 49(2):467-475, 2000.

[2] S. S. Saab. A map matching approach for train positioning part II application and experimentation. IEEE Transactions on Vehicular Technology, 49(2):476-484, 2000.

[3] J. Holland. Adaptation in Natural and Artificial Systems. 1975.

[4] J. Kennedy and R. C. Eberhart. Particle swarm optimization. In Proceedings of IEEE International Conference on Neural Networks, volume 4, pages 1942-1948, 1995.

[5] R. C. Eberhart and J. Kennedy. A new optimizer using particle swarm theory. In Proceedings of the Sixth International Symposium on Micro Machine and Human Science, pages 39-43, 1995.

[6] M. Dorigo. Optimization, Learning and Natural Algorithms. PhD thesis, Politecnico di Milano, Italy, 1992.

[7] S. Kirkpatrick, C. D. Gelatt, and M. P. Vecchi. Optimization by simulated annealing. Science, 220(4598):671-680, 1983.

[8] X. S. Yang. Firefly algorithms for multimodal optimization. In Proceedings of 5th International Symposium on Stochastic Algorithms: Foundations and Applications, pages 169-178, 2009.

[9] R. G. Reynolds. An introduction to cultural algorithms. In Proceedings of the 3rd Annual Conference on Evolutionary Programming, pages 131-139, 1994.

[10] S. H. Hamed. The intelligent water drops algorithm: a nature-inspired swarm-based optimization algorithm. International Journal of Bio-Inspired Computation, 1(1/2):2009, 7179.
[11] A. Kaveh and S. Talatahari. A novel heuristic optimization method: charged system search. Acta Mechanica, 213(3):2010, 267-289.

[12] A. H. Gandomi and A. H. Alavi. Krill herd: A new bio-inspired optimization algorithm. Communications in Nonlinear Science and Numerical Simulation, 17(12):2010, 4831-4845.

[13] D. Karaboga. An idea based on honey bee swarm for numerical optimization. Technical Report-TR06, Erciyes Univeristy, page 2005.

[14] X. S. Yang and S. Deb. Cuckoo search via levy fights. In Proceedings of IEEE world congress on Nature and Biologically Inspired Computing, pages 210-214, 2009.

[15] Y. Shi and R. C. Eberhart. A modified particle swarm optimizer. In Proceedings of IEEE World Congress on Computational Intelligence, pages 69-73, 1998.

[16] I. C. Trelea. The particle swarm optimization algorithm: convergence analysis and parameter selection. Information Processing Letters, 85(6):317-325, 2003.

[17] M. Clerc and J. Kennedy. The particle swarm explosion, stability, and convergence in a multidimensional complex space. IEEE Transactions on Evolutionary Computation, 6(1):5873, 2002.

[18] L. F. M. Juan and G. G. Esperanza. Stochastic stability analysis of the linear continuous and discrete PSO models. IEEE Transactions on Evolutionary Computation, 15(3):405-423, 2011.

[19] R. Storn and K. Price. Differential evolution a simple and efficient heuristic for global optimization over continuous spaces. Journal of Global Optimization, 11(4):341-359, December 1997.

[20] D. Karaboga and B. Basturk. A powerful and efficient algorithm for numerical function optimization: artificial bee colony (ABC) 
algorithm. Journal of Global Optimization, 39(3):459-471, November 2007.

[21] X. S. Yang and S. Deb. Cuckoo search via levy flights. In Proceedings of 2009 World Congress on Nature Biologically Inspired Computing, pages 210-214, 2009.

[22] S. He, Q. H. Wu, and J. R. Saunders. Group search optimizer: An optimization algorithm inspired by animal searching behavior. IEEE Transactions on Evolutionary Computation, 13(5):973-990, 2009.

[23] X. S. Yang. Firefly algorithms for multimodal optimization. In Proceedings of 5th Interna- tional Symposium on Stochastic Algorithms: Foundations and Applications, pages 169-178, 2009.

[24] Brian L. Partridge. Structure and function of fish schools. Scientific American, 246(6):114123, 1982.

[25] E. Shaw. Fish in school. Natural History, 84(8):40-45, 1975.

[26] R. C. Eberhart and Y. Shi. Comparing inertia weights and constriction factors in particle swarm optimization. In Proceedings of the 2000 Congress on Evolutionary Computation, pages 84-88, 2000. 
Table 1: The initial position and velocity of the swarm

\begin{tabular}{|c|c|c|}
\hline Function & Initial Position & Initial Velocity \\
\hline Sphere & $(50,100)^{D}$ & $(-50,50)^{D}$ \\
\hline Griewank & $(300,600)^{D}$ & $(-300,300)^{D}$ \\
\hline Rastrigin & $(2.56,5.12)^{D}$ & $(-2.56,2.56)^{D}$ \\
\hline Rosenbrock & $(15,30)^{D}$ & $(-15,15)^{D}$ \\
\hline Schaffer's f6 & $(50,100)^{D}$ & $(-50,50)^{D}$ \\
\hline
\end{tabular}

Table 2: Results on the modified Sphere function from 2 dimensions to 20 dimensions

\begin{tabular}{|c|c|c|c|c|c|}
\hline Dim & {$[\mathbf{0 , 0 . 0 1}]$} & {$[\mathbf{0 , 0 . 0 2}]$} & {$[\mathbf{0 , 0 . 0 3}]$} & {$[\mathbf{0 , 0 . 0 4}]$} & {$[\mathbf{0 , 0 . 0 5}]$} \\
\hline 2 & 0.0127 & $2.29 \mathrm{E}-4$ & $2.73 \mathrm{E}-6$ & $2.73 \mathrm{E}-8$ & $3.1 \mathrm{E}-10$ \\
\hline 4 & 0.9404 & 0.0095 & $9.34 \mathrm{E}-5$ & $7.66 \mathrm{E}-7$ & $8.52 \mathrm{E}-9$ \\
\hline 6 & 4.2850 & 0.0370 & $3.65 \mathrm{E}-4$ & $3.37 \mathrm{E}-6$ & $3.22 \mathrm{E}-8$ \\
\hline 8 & 9.3113 & 0.0795 & $8.18 \mathrm{E}-4$ & $6.80 \mathrm{E}-6$ & $6.04 \mathrm{E}-8$ \\
\hline 10 & 15.096 & 0.1408 & 0.0013 & $1.08 \mathrm{E}-5$ & $1.11 \mathrm{E}-7$ \\
\hline 12 & 22.808 & 0.1936 & 0.0018 & $1.73 \mathrm{E}-5$ & $1.60 \mathrm{E}-7$ \\
\hline 14 & 29.961 & 0.2828 & 0.0027 & $2.33 \mathrm{E}-5$ & $2.30 \mathrm{E}-7$ \\
\hline 16 & 40.518 & 0.3625 & 0.0034 & $3.18 \mathrm{E}-5$ & $3.12 \mathrm{E}-7$ \\
\hline 18 & 47.777 & 0.4515 & 0.0046 & $4.26 \mathrm{E}-5$ & $4.14 \mathrm{E}-7$ \\
\hline 20 & 58.320 & 0.5491 & 0.0055 & $5.15 \mathrm{E}-5$ & $5.52 \mathrm{E}-7$ \\
\hline
\end{tabular}

Table 3: Results on Griewank function from 2 dimensions to 20 dimensions

\begin{tabular}{|c|c|c|c|c|c|}
\hline Dim & {$[\mathbf{0 , 0 . 0 1}]$} & {$[\mathbf{0 , 0 . 0 2}]$} & {$[\mathbf{0 , 0 . 0 3}]$} & {$[\mathbf{0 , 0 . 0 4}]$} & {$[\mathbf{0 , 0 . 0 5}]$} \\
\hline 2 & 0.0256 & 0.0061 & 0.0039 & 0.0034 & 0.0043 \\
\hline 4 & 0.2614 & 0.1018 & 0.0919 & 0.0828 & 0.0660 \\
\hline 6 & 0.6257 & 0.2889 & 0.2660 & 0.2569 & 0.2350 \\
\hline 8 & 0.9329 & 0.4929 & 0.4725 & 0.4088 & 0.3550 \\
\hline 10 & 1.1021 & 0.6841 & 0.5082 & 0.4319 & 0.3538 \\
\hline 12 & 1.2037 & 0.7389 & 0.3968 & 0.2661 & 0.1489 \\
\hline 14 & 1.2917 & 0.7966 & 0.3604 & 0.1770 & 0.0968 \\
\hline 16 & 1.3700 & 0.8231 & 0.3820 & 0.1977 & 0.1069 \\
\hline 18 & 1.4526 & 0.8636 & 0.3981 & 0.1987 & 0.1134 \\
\hline 20 & 1.5552 & 0.8692 & 0.3842 & 0.2083 & 0.1078 \\
\hline
\end{tabular}


Table 4: Results on Rastrigin function from 2 dimensions to 20 dimensions

\begin{tabular}{|c|c|c|c|c|c|}
\hline Dim & {$[\mathbf{0 , 0 . 0 1}]$} & {$[\mathbf{0 , 0 . 0 2}]$} & {$[\mathbf{0 , 0 . 0 3}]$} & {$[\mathbf{0 , 0 . 0 4}]$} & {$[\mathbf{0 , 0 . 0 5}]$} \\
\hline 2 & 0.0081 & $3.09 \mathrm{E}-4$ & $1.19 \mathrm{E}-5$ & $2.57 \mathrm{E}-5$ & $8.15 \mathrm{E}-5$ \\
\hline 4 & 1.9545 & 1.6540 & 1.9113 & 1.5498 & 1.7123 \\
\hline 6 & 7.8926 & 6.4301 & 6.8185 & 6.9780 & 6.8022 \\
\hline 8 & 17.385 & 15.449 & 15.105 & 15.196 & 15.689 \\
\hline 10 & 29.899 & 25.583 & 26.161 & 25.114 & 24.812 \\
\hline 12 & 44.793 & 37.976 & 38.124 & 38.796 & 33.088 \\
\hline 14 & 59.219 & 50.824 & 52.300 & 48.108 & 42.097 \\
\hline 16 & 71.023 & 65.924 & 61.875 & 56.151 & 62.952 \\
\hline 18 & 90.020 & 83.083 & 68.056 & 76.806 & 72.217 \\
\hline 20 & 107.08 & 90.782 & 91.178 & 87.137 & 85.103 \\
\hline
\end{tabular}

Table 5: Results on Rosenbrock function from 2 dimensions to 20 dimensions

\begin{tabular}{|c|c|c|c|c|c|}
\hline Dim & {$[\mathbf{0 , 0 . 0 1}]$} & {$[\mathbf{0 , 0 . 0 2}]$} & {$[\mathbf{0 , 0 . 0 3}]$} & {$[\mathbf{0 , 0 . 0 4}]$} & {$[\mathbf{0 , 0 . 0 5}]$} \\
\hline 2 & 1.9650 & 1.4854 & 1.5935 & 1.0477 & 2.2424 \\
\hline 4 & 35.500 & 12.136 & 8.6936 & 6.4596 & 8.7288 \\
\hline 6 & 197.05 & 147.97 & 214.63 & 197.13 & 55.260 \\
\hline 8 & 528.20 & 395.20 & 237.93 & 433.56 & 622.21 \\
\hline 10 & 821.85 & 527.42 & 554.99 & 390.86 & 669.12 \\
\hline 12 & 1172.0 & 419.49 & 616.79 & 602.11 & 739.81 \\
\hline 14 & 1260.5 & 782.67 & 973.57 & 499.15 & 686.13 \\
\hline 16 & 1324.7 & 600.75 & 350.37 & 674.49 & 1098.5 \\
\hline 18 & 1847.2 & 760.68 & 881.50 & 837.81 & 955.85 \\
\hline 20 & 2107.4 & 694.28 & 636.72 & 976.27 & 877.51 \\
\hline
\end{tabular}

Table 6: Results on Schaffer's f6 function under the 2-dimensional space

\begin{tabular}{|c|c|c|c|c|c|}
\hline Dim & {$[\mathbf{0 , 0 . 0 1}]$} & {$[\mathbf{0 , 0 . 0 2}]$} & {$[\mathbf{0 , 0 . 0 3}]$} & {$[\mathbf{0 , 0 . 0 4}]$} & {$[\mathbf{0 , 0 . 0 5}]$} \\
\hline 2 & 0.0083 & 0.0030 & 0.0031 & 0.0027 & 0.0020 \\
\hline
\end{tabular}

Table 7: Results on Griewank function from 3 dimensions to 30 dimensions

\begin{tabular}{|c|c|c|c|}
\hline Dim & ECO & PSO-IW & PSO-CF \\
\hline 3 & 0.0270 & 0.0197 & 0.0220 \\
\hline 6 & 0.2074 & 0.1082 & 0.1054 \\
\hline 9 & 0.4055 & 0.1303 & 0.1549 \\
\hline 12 & 0.1678 & 0.1091 & 0.1277 \\
\hline 15 & 0.0833 & 0.0608 & 0.2078 \\
\hline 18 & 0.0958 & 0.0844 & 1.1063 \\
\hline 21 & 0.1029 & 0.2248 & 1.1440 \\
\hline 24 & 0.1082 & 0.7185 & 1.3068 \\
\hline 27 & 0.1185 & 1.7553 & 2.1678 \\
\hline 30 & 0.1254 & 1.8506 & 4.5117 \\
\hline
\end{tabular}


Table 8: Results on Rastrigin function from 3 dimensions to 30 dimensions

\begin{tabular}{|c|c|c|c|}
\hline Dim & ECO & PSO-IW & PSO-CF \\
\hline 3 & 0.4337 & 0.1393 & 1.2934 \\
\hline 6 & 7.6859 & 2.5118 & 10.447 \\
\hline 9 & 16.758 & 8.4354 & 27.421 \\
\hline 12 & 30.001 & 18.090 & 38.525 \\
\hline 15 & 42.599 & 35.641 & 64.911 \\
\hline 18 & 60.289 & 50.217 & 87.775 \\
\hline 21 & 87.719 & 79.982 & 117.34 \\
\hline 24 & 94.571 & 98.361 & 154.72 \\
\hline 27 & 105.58 & 138.22 & 182.23 \\
\hline 30 & 123.83 & 152.42 & 207.44 \\
\hline
\end{tabular}

Table 9: Results on Schaffer's f6 function under the 2-dimensional space

\begin{tabular}{|c|c|c|c|}
\hline Dim & ECO & PSO-IW & PSO-CF \\
\hline 2 & 0.0024 & 0.0066 & 0.0066 \\
\hline
\end{tabular}

